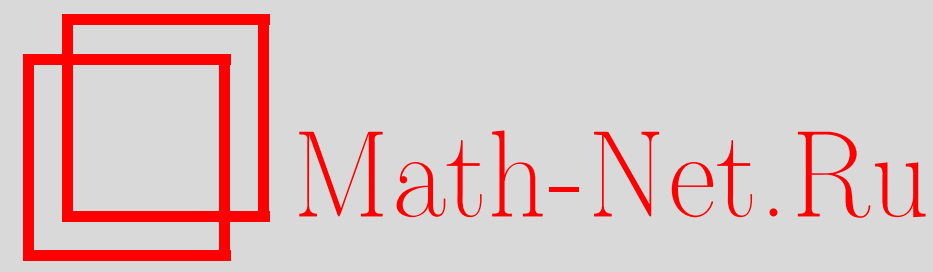

М. И. Вишик, В. В. Чепыжов, Усреднение траекторных аттракторов эволюционных уравнений с быстро осциллирующими членами, Матем. сб., 2001, том 192, номер 1, 13-50

DOI: https://doi.org/10.4213/sm534

Использование Общероссийского математического портала Math-Net.Ru подразумевает, что вы прочитали и согласны с пользовательским соглашением

http://www . mathnet.ru/rus/agreement

Параметры загрузки:

IP : 3.89 .197 .203

26 апреля 2023 г., 12:34:52 


\author{
М.И. Вишик, В.В. Чепьжов
}

\title{
Усреднение траекторных аттракторов эволюционных уравнений с быстро осциллирующими членами
}

\begin{abstract}
Рассматриваются эволюционные уравнения, которые содержат члены, быстро осциллирующие по пространственньм переменным или по переменной времени. Доказано, что в пределе траекторные аттракторы этих уравнений стремятся к траек торньп аттракторам уравнений, члены которых являются средними соответствующих быстро осциллирующих членов исходных уравнений. При этом не предполагается однозначная разрешимость соответствуюших задач Коши. Если задачи Коши для рассматриваемых уравнений однозначно разрешимы, то они порождают полугрупшы, имеющие глобальные аттракторы. Эти аттракторы также сходятся к глобальным аттракторам усредненных уравнений в соответствующих пространствах.

В качестве примеров рассмотрены следующие уравнения и системы математической физики: трехмерная и двумерная системы Навье-Стокса с быстро осциллирующими внешними силами, системы уравнений реакции-диффузии, комплексное уравнение Гинзбурга-Ландау, обобщенное уравнение Чэфи-Инфанте, диссипативные гиперболические уравнения с быстро осциллирующими членами и коэффициентами.

Библиография: 20 названий.
\end{abstract}

\section{Введение}

В настоящей статье исследуются аттракторы эволюционных уравнений, содержащих быстро осциллирующие члены по пространственным и временной переменньм. Частота осцилляции характеризуется параметром $\varepsilon>0$. Наряду с таким уравнением рассматривается соответствующее ему усредненное уравнение. Для основных диссипативных уравнений математической физики доказано, что траекторный аттрактор $\mathfrak{A}_{\varepsilon}$ уравнения с осциллируюшими членами сходится при $\varepsilon \rightarrow 0+$ к траекторному аттрактору $\overline{\mathfrak{A}}$ усредненного уравнения в соответствуюшем функциональном пространстве. При этом не предполагается, что задача Коши для этого уравнения обладает единственным решением. Так, например, для трехмерной системы уравнений Навье-Стокса с внешней силой $g(x, x / \varepsilon)$ доказано, что ее траекторный аттрактор $\mathfrak{A}_{\varepsilon}$ стремится при $\varepsilon \rightarrow 0+$ к траекторному аттрактору $\overline{\mathfrak{A}}$ системы Навье-Стокса с внешней силой $\bar{g}(x)$, где $\bar{g}(x)$ - усреднение $g(x, x / \varepsilon)$ при $\varepsilon \rightarrow 0+$, например, в пространстве $H_{w}\left(H_{w}\right.$ - пространство $H$, снабженное слабой топологией). Аналогичные теоремы доказаны для систем уравнений реакции-диффузии, содержащих, например, члены вида $b(x, x / \varepsilon) f(u)$ и $g(x, x / \varepsilon)$, а также для диссипативных гиперболических уравнений с осциллирующими членами и для других уравнений. В том случае, когда для рассматриваемого уравнения $\mathrm{c}$

Работа выполнена при частичной поддержке Российского фонда фундаментальных исследований (грант № 99-01-00304). 
быстро осциллирующими членами имеет место теорема единственности решения задачи Коши и выполнены некоторые естественные условия, доказано, что глобальньй аттрактор $\mathscr{A}_{\varepsilon}$ такого уравнения (или системы) сходится в соответствующем функциональном пространстве при $\varepsilon \rightarrow 0+$ к глобальному аттрактору $\overline{\mathscr{A}}$ усредненного уравнения. При этом сушественно используется тот факт, что траекторные аттракторы $\mathfrak{A}_{\varepsilon}$ сходятся к траекторному аттрактору $\overline{\mathfrak{A}}$ усредненного уравнения.

Некоторые проблемы, связанные со сходимостью аттракторов $\mathscr{A}_{\varepsilon} \mathrm{k} \overline{\mathscr{A}}$ для уравнений с быстро осциллирующими по времени членами, исследовались в [1], [2] и [3].

В $\S 1$ излагаются некоторые вопросы, связанные с усреднением быстро осциллирующих функций. В $\S 2$ даются основные сведения о траекторных аттракторах эволюционных уравнений. Проблеме усреднения аттракторов диссипативных уравнений математической физики с быстро осциллирующими членами посвящен $\S 3$.

\section{§ 1. Усреднение быстро осциллирующих функций}

В этом параграфе мы кратко рассмотрим некоторые вопросы, связанные с быстро осциллирующими функциями. Прежде всего мы изучим усреднение функций вида $b(x / \varepsilon)$ и $b(x, x / \varepsilon)$ при $\varepsilon \rightarrow 0+$. Переменную $z=x / \varepsilon$ мы будем называть "быстрой", а переменную $x$ - "медленной". Здесь $z$ и $x$ являются пространственньми переменными, т.е. $z \in \mathbb{R}^{n}$ и $x \in \Omega \Subset \mathbb{R}^{n}$. Позднее мы также будем исследовать усреднение по времени функций вида $b(x, t / \varepsilon)$ и $b(x, t, t / \varepsilon)$ при $\varepsilon \rightarrow 0+$, где $x \in \Omega \Subset \mathbb{R}^{n}$ и $t \in \mathbb{R}$. Заметим, что аналогичными методами можно изучать усреднение более сложных функций вида $b(x, x / \varepsilon, t, t / \varepsilon)$.

1.1. Пространственное усреднение. Рассматривается семейство вешественных функций $\left\{f_{\varepsilon}(x), \varepsilon>0\right\}$ в области $\Omega \Subset \mathbb{R}^{n}$, зависящее от малого положительного параметра $\varepsilon$. Пусть $f_{\varepsilon}(x) \in L_{p}(\Omega)$, где $p>1$. Будем говорить, что функции $f_{\varepsilon}(x)$ имеют среднее $\bar{f}(x) \in L_{p}(\Omega)$ при $\varepsilon \rightarrow 0+$ в пространстве $L_{p, w}(\Omega)$, если

$$
f_{\varepsilon}(x) \neg \bar{f}(x) \text { при } \varepsilon \rightarrow 0+\text { слабо в } L_{p}(\Omega),
$$

т.е. для любой функции $\varphi(x) \in L_{q}(\Omega), 1 / p+1 / q=1$,

$$
\int_{\Omega} f_{\varepsilon}(x) \varphi(x) d x \rightarrow \int_{\Omega} \bar{f}(x) \varphi(x) d x \quad \text { при } \varepsilon \rightarrow 0+.
$$

Аналогичным образом определяется среднее для семейства функций $f_{\varepsilon}(x) \in L_{\infty}(\Omega)$ при $\varepsilon \rightarrow 0+$ в пространстве $L_{\infty, * w}(\Omega)$. В этом случае среднее $\bar{f}(x) \in L_{\infty}(\Omega)$ и условие (1.2) должно быть выполнено для любой функции $\varphi(x) \in L_{1}(\Omega)\left(L_{\infty}(\Omega)=\right.$ $\left.L_{1}(\Omega)^{*}\right)$, т.е. $f_{\varepsilon}(x) \rightarrow \bar{f}(x)$ при $\varepsilon \rightarrow 0+*$-слабо в $L_{\infty}(\Omega)$. Если функции $f_{\varepsilon}(x)$ имеют среднее $\bar{f}(x)$ в пространстве $L_{\infty, * w}(\Omega)$, то, очевидно, они имеют то же среднее в пространстве $L_{p, w}(\Omega)$ при любом $p>1$.

Далее будут изучаться функции вида $b(x, x / \varepsilon)$. Для простоты предполагается, что $0 \in \Omega$ и область $\Omega$ является звездной, т.е. $\Omega \subseteq \lambda \Omega$ для любого числа $\lambda \geqslant 1$.

Пусть задана вещественная функция $b(x, z), x \in \Omega \Subset \mathbb{R}^{n}, z \in \mathbb{R}^{n}$. Во многих случаях можно определить функцию $f_{\varepsilon}(x)=b(x, x / \varepsilon)$. Например, если $b(x, z) \in$ $C\left(\bar{\Omega} \times \mathbb{R}^{n}\right)$, то функция $b(x, x / \varepsilon)$ является корректно определенной при $x \in \Omega$. В дальнейшем будут приведены другие примеры таких функций. Изучается усреднение функций $b(x, x / \varepsilon)$ при $\varepsilon \rightarrow 0+$ в пространствах $L_{p, w}(\Omega)$ и $L_{\infty, * w}(\Omega)$. 
Сначала рассмотрим функции вида $b(x / \varepsilon)$, где $b(z) \in L_{\infty}^{\text {loc }}\left(\mathbb{R}^{n}\right)$ или $b(z) \in$ $L_{p}^{\text {loc }}\left(\mathbb{R}^{n}\right)$ для некоторого $p>1$. Рассмотрим подробнее случай, когда $\bar{b}(x) \equiv$ const.

Обозначим через $P_{a_{1} \ldots a_{n}}^{b_{1} \ldots b_{n}}=\left[a_{1}, b_{1}\right] \times \cdots \times\left[a_{n}, b_{n}\right]=P$ параллелепипед в $\mathbb{R}^{n}$ объема $|P|=\prod_{i=1}^{n}\left(b_{i}-a_{i}\right)$. Предположим, что функция $b(x / \varepsilon)$ имеет среднее $\bar{b} \in \mathbb{R}$ в $L_{p, w}(\Omega)$. Мы утверждаем, что для любого параллелепипеда $P_{a_{1} \ldots a_{n}}^{b_{1} \ldots b_{n}}=P$ вьполнено:

$$
\frac{1}{|\lambda P|} \int_{\lambda P} b(z) d z \rightarrow \bar{b} \text { при } \lambda \rightarrow+\infty .
$$

Здесь $|\lambda P|=\lambda^{n}|P|=\lambda^{n} \prod_{i=1}^{n}\left(b_{i}-a_{i}\right)$. В самом деле, положим в $(1.2) \varphi(x)=$ $\chi_{P}(x)$, где $\chi_{P}(x)$ - характеристическая функция $P$, т.е. $\chi_{P}(x)=1$, если $x \in P$, и $\chi_{P}(x)=0$ в противном случае. Тогда из (1.2) для $\lambda=1 / \varepsilon$ вытекает, что

$$
\int_{P} b(\lambda x) d x \rightarrow \int_{P} \bar{b} d x=|P| \bar{b} \text { при } \lambda \rightarrow+\infty .
$$

Сделав замену переменной $\lambda x=z$ в левой части (1.4), получаем

$$
\frac{1}{\lambda^{n}} \int_{\lambda P} b(z) d z \rightarrow|P| \bar{b} \text { при } \lambda \rightarrow+\infty,
$$

и (1.3) доказано. Обратное утверждение также верно. Имеет место следующий критерий.

ТЕОРема 1.1. Пусть $b(z) \in L_{p}^{\text {loc }}\left(\mathbb{R}^{n}\right)$. Функиия $b(x / \varepsilon)$ имеет среднее $\bar{b} \in \mathbb{R}$ в $L_{p, w}(\Omega)$ тогда и только тогда, если

(i) семейство $\{b(x / \varepsilon), 1>\varepsilon>0\}$ ограничено в $L_{p}(\Omega)$;

(ii) предел (1.3) имеет место для каждого параллелепипеда $P_{a_{1} \ldots a_{n}}^{b_{1} \ldots b_{n}}$ такого, что $a_{1} b_{1}=\cdots=a_{n} b_{n}=0$. (Это условие означает, что одна из вериин параллелепипеда $P$ находится в начале координат $\mathbb{R}^{n}$.)

ДокАЗАТЕльство. Необходимость (i) очевидна, а необходимость (ii) была доказана выше. Докажем обратное утверждение. В силу того, что семейство $\{b(x / \varepsilon)$, $1>\varepsilon>0\}$ ограничено в $L_{p}(\Omega)$, достаточно доказать, что

$$
\int_{\Omega} b\left(\frac{x}{\varepsilon}\right) \varphi(x) d x \rightarrow \bar{b} \int_{\Omega} \varphi(x) d x \quad \text { при } \varepsilon \rightarrow 0+
$$

для любой функции $\varphi(x)$, принадлежащей всюду плотному множеству из $L_{q}(\Omega)$. Мы докажем это утверждение для множества ступенчатых функций, которые являются линейными комбинациями характеристических функций параллелепипедов в $\mathbb{R}^{n}$, т.е. для функций $\varphi$ вида $\varphi(x)=\sum_{j=1}^{N} \alpha_{j} \chi_{P_{j}}(x)$, где $P_{j}=P_{a_{1}(j) \ldots a_{n}(j)}^{b_{1}(j) \ldots b_{n}(j)}$. (Множество этих функций всюду плотно в $L_{q}(\Omega)$.) Необходимо доказать $(1.5)$ для характеристической функции $\chi_{P}(x)$ любого параллелепипеда $P_{a_{1} \ldots a_{n}}^{b_{1} \ldots b_{n}}$. Заметим, наконец, что любой параллелепипед $P_{a_{1} \ldots a_{n}}^{b_{1} \ldots b_{n}}$ может быть разбит на параллелепипеды, которые удовлетворяют условию (ii), и, следовательно, характеристическая функция $P_{a_{1} \ldots a_{n}}^{b_{1} \ldots b_{n}}$ является линейной комбинацией этих характеристических функций. Например, если $n=2$ и $0<a_{1}<b_{1}, 0<a_{2}<b_{2}$, то

$$
\chi_{P_{a_{1} a_{2}}^{b_{1} b_{2}}}=\chi_{P_{00}^{b_{1} b_{2}}}-\chi_{P_{00}^{a_{1} b_{2}}}-\chi_{P_{00}^{a_{2} b_{1}}}+\chi_{P_{00}^{a_{1} a_{2}}} .
$$

Для таких характеристических функций условие (1.5) эквивалентно условию (1.3), которое выполнено в силу предположения (ii). Теорема доказана. 
ЗАмЕчАниЕ 1.1. Свойство (i) выполнено, например, если функция $b(z)$ равномерно ограничена в $L_{p}^{\text {loc }}\left(\mathbb{R}^{n}\right)$ в следующем смысле:

$$
\sup _{z_{0} \in \mathbb{R}^{n}} \int_{P_{0 \ldots 0}^{1 \ldots 1}}\left|f\left(z+z_{0}\right)\right|^{p} d z<+\infty,
$$

где $P_{0 \ldots 0}^{1 \ldots 1}-$ единичный куб $[0,1]^{n}$ в $\mathbb{R}^{n}$.

Аналогично доказывается следующая

Теорема 1.2. Пусть $b(z) \in L_{\infty}^{\text {loc }}\left(\mathbb{R}^{n}\right)$. Функиия $b(x / \varepsilon)$ имеет среднее $\bar{b} \in \mathbb{R}$ в $L_{\infty, * w}(\Omega)$, если и только если

(i) $b(z) \in L_{\infty}\left(\mathbb{R}^{n}\right)$;

(ii) предел (1.3) имеет место для любого параллелепипеда $P_{a_{1} \ldots a_{n}}^{b_{1} \ldots b_{n}}$ такого, что $a_{1} b_{1}=\cdots=a_{n} b_{n}=0$.

Сформулируем еще одно достаточное условие усредняемости. Обозначим через $K_{\lambda}\left(x_{0}\right)$ куб в $\mathbb{R}^{n}$ с центром $x_{0}$ и ребром $\lambda$ :

$$
K_{\lambda}\left(x_{0}\right)=\left\{\left|x_{j}-x_{0 j}\right| \leqslant \frac{\lambda}{2}, j=1, \ldots, n\right\} .
$$

Tеорема 1.3. Пусть $b(z) \in L_{\infty}(\Omega)$. Обозначим

$$
b_{\lambda}\left(x_{0}\right)=\frac{1}{\lambda^{n}} \int_{K_{\lambda}\left(x_{0}\right)} b(z) d z .
$$

Предположим, что найдется число $\bar{b}$ такое, что для всех $\lambda>0$ и $x_{0} \in \mathbb{R}^{n}$

$$
\left|b_{\lambda}\left(x_{0}\right)-\bar{b}\right| \leqslant \beta\left(\frac{1}{\lambda}\right)
$$

где $\beta(s) \rightarrow 0$ при $s \rightarrow 0+$. Тогда для каждой области $\Omega \Subset \mathbb{R}^{n}$ функиия $b(x / \varepsilon)$ имеет среднее $\bar{b}$ при $\varepsilon \rightarrow 0+$ в $L_{\infty, * w}(\Omega)$.

Мы опускаем доказательство, которое заключается в проверке того, что соотношение (1.7) влечет вьполнение условия (ii) теоремы 1.2.

Теперь рассмотрим усреднение функций, зависящих как от быстрых, так и от медленных переменных вида $b(x, x / \varepsilon), x \in \Omega \Subset \mathbb{R}^{n}$.

ПрИмеР 1.1. Пусть $b(x, x / \varepsilon)=b_{1}(x) b_{2}(x / \varepsilon)$, где $b_{1}(x) \in L_{p}(\Omega)$ и $b_{2}(z) \in$ $L_{\infty}\left(\mathbb{R}^{n}\right)$. Предположим, что функция $b_{2}(x / \varepsilon)$ имеет среднее $\bar{b}_{2} \in \mathbb{R}$ в $L_{\infty, * w}(\Omega)$. Тогда функция $b(x, x / \varepsilon)$, очевидно, имеет среднее $\bar{b}(x)=b_{1}(x) \bar{b}_{2}$ в $L_{p, w}(\Omega)$.

Аналогичньм образом обладают усреднением функции вида

$$
b^{N}\left(x, \frac{x}{\varepsilon}\right)=\sum_{i=1}^{N} b_{1 i}(x) b_{2 i}\left(\frac{x}{\varepsilon}\right)
$$

где $b_{1 i}(x) \in L_{p}(\Omega), b_{2 i}(z) \in L_{\infty}\left(\mathbb{R}^{n}\right)$ и функции $b_{2 i}(x / \varepsilon)$ имеют средние $\bar{b}_{2 i} \in \mathbb{R}$ в $L_{\infty, * w}(\Omega)$. 
ПримеР 1.2. Пусть дана функция $b(x, z) \in C\left(\bar{\Omega} \times \mathbb{R}^{n}\right)$, которая является квазипериодической по каждому аргументу $z_{j}, j=1, \ldots, n\left(z=\left(z_{1}, \ldots, z_{n}\right)\right)$. Точнее, имеется непрерывная функция

$$
B\left(x, \omega_{11}, \ldots, \omega_{1 k_{1}}, \ldots, \omega_{n 1}, \ldots, \omega_{n k_{n}}\right) \in C\left(\bar{\Omega} \times \mathbb{T}^{k_{1}} \times \cdots \times \mathbb{T}^{k_{n}}\right),
$$

которая является 1-периодической по каждому аргументу $\omega_{i j}$ и такая, что

$$
b\left(x, z_{1}, \ldots, z_{n}\right)=B\left(x, \alpha_{11} z_{1}, \ldots, \alpha_{1 k_{1}} z_{1}, \ldots, \alpha_{n 1} z_{n}, \ldots, \alpha_{n k_{n}} z_{n}\right) \quad \forall z \in \mathbb{R}^{n}
$$

Здесь $\left\{\alpha_{i j}\right\}_{j=1, \ldots, k_{i}}^{i=1, \ldots, n}-$ рационально независимые вещественные числа.

Множество $\bar{\Omega} \times \mathbb{T}^{k_{1}} \times \cdots \times \mathbb{T}^{k_{n}}$ компактно. Поэтому функция $b(x, z)$ равномерно непрерывна по $x$ :

$$
\left|b\left(x_{1}, z\right)-b\left(x_{2}, z\right)\right| \leqslant \alpha\left(\left|x_{1}-x_{2}\right|\right) \quad \forall x_{1}, x_{2} \in \bar{\Omega}, \quad \forall z \in \mathbb{R}^{n},
$$

где $\alpha(s) \rightarrow 0(s \rightarrow 0+)$ и $\alpha(s)$ не зависит от $z$.

Используя теорему Кронекера-Вейля и теорему об однопараметрическом семействе трансляций на торе (см. [4]), можно доказать, что для каждой точки $z^{0}=\left(z_{1}^{0}, \ldots, z_{n}^{0}\right) \in \mathbb{R}^{n}$

$$
\begin{aligned}
& \frac{1}{\lambda_{1} \ldots \lambda_{n}} \int_{z_{1}^{0}}^{z_{1}^{0}+\lambda_{1}} \ldots \int_{z_{n}^{0}}^{z_{n}^{0}+\lambda_{n}} b\left(x, z_{1}, \ldots, z_{n}\right) d z_{1} \ldots d z_{n} \\
& \quad \rightarrow \int_{\mathbb{T}^{k_{1}}} \cdots \int_{\mathbb{T}^{k_{n}}} B\left(x, \bar{\omega}_{1}, \ldots, \bar{\omega}_{n}\right) \mu\left(d \bar{\omega}_{1} \ldots d \bar{\omega}_{n}\right) \equiv \bar{b}(x) \quad\left(\lambda_{i} \rightarrow+\infty\right) .
\end{aligned}
$$

Здесь $\bar{\omega}_{i}=\left(\omega_{i 1}, \ldots, \omega_{i k_{i}}\right) \in \mathbb{T}^{k_{i}}, i=1, \ldots, n, \mu\left(d \omega_{1} \ldots d \omega_{n}\right)$ - мера Лебега на $\mathbb{T}^{K}=\mathbb{T}^{k_{1}} \times \cdots \times \mathbb{T}^{k_{n}}, K=k_{1}+\cdots+k_{n}$, и $\mu\left(\mathbb{T}^{K}\right)=1$. Предельное соотношение (1.11) вьполнено равномерно по $z^{0} \in \mathbb{R}^{n}$ и по $x \in \bar{\Omega}$. Используя (1.11) и (1.10), легко показать, что функция $\bar{b}(x)$ является средним для $b(x, x / \varepsilon)$ в $L_{\infty, * w}(\Omega)$ при $\varepsilon \rightarrow 0+$. В частности, из (1.11) следует, что

$$
\left|\frac{1}{\lambda^{n}} \int_{K_{\lambda}\left(x_{0}\right)} b\left(x_{0}, z\right) d z-\bar{b}\left(x_{0}\right)\right| \leqslant \beta\left(\frac{1}{\lambda}\right)
$$

где $\beta(s) \rightarrow 0(s \rightarrow 0+)$ и $\beta(s)$ не зависит от $x_{0}$.

Наконец, отметим, что условие (1.12) влечет следующий результат об усреднении, обобщающий теорему 1.3.

Tеорема 1.4. Пусть $b(x, z) \in C_{b}\left(\bar{\Omega} \times \mathbb{R}^{n}\right), \bar{b}(x) \in L_{\infty}(\Omega)$. Предположимм, что выполнены свойства (1.10) и (1.12). Тогда функиия $b(x, x / \varepsilon)$ имеет среднее $\bar{b}(x)$ в $L_{\infty, * w}(\Omega)$ при $\varepsilon \rightarrow 0+$. 
ПРИмеР 1.3. Рассмотрим функцию $b(x, z) \in C_{b}\left(\bar{\Omega} \times \mathbb{R}^{n}\right)$, удовлетворяющую условию (1.10). Пусть также функция $b(x, z)$ является почти периодической по $z$ в смысле Бора, т.е. существуют квазипериодические функции $b_{N}(x, z) \in C_{b}\left(\bar{\Omega} \times \mathbb{R}^{n}\right)$ (см. (1.9)), которые удовлетворяют (1.10) с одной и той же функцией $\alpha(s)$ и такие, что

$$
\lim _{N \rightarrow \infty}\left\|b(x, z)-b_{N}(x, z)\right\|_{C_{b}\left(\bar{\Omega} \times \mathbb{R}^{n}\right)}=0
$$

(см. [5]). При выполнении перечисленных условий функция $b(x, x / \varepsilon)$ имеет среднее $\bar{b}(x)$ в $L_{\infty, * w}(\Omega)$ при $\varepsilon \rightarrow 0+$, где $\bar{b}(x)=\lim _{N \rightarrow \infty} \bar{b}_{N}(x)$ и $\bar{b}_{N}(x)-$ средние функций $b_{N}(x, z)$ в $L_{\infty, * w}(\Omega)($ см. $(1.11))$.

1.2. Усреднение по времени. Рассмотрим усреднение по времени функций вида $g(x, t / \varepsilon)$ и $g(x, t, t / \varepsilon)$, где $t \in \mathbb{R}$ и $x \in \Omega$.

Пусть $H$ обозначает сепарабельное гильбертово (или банахово) пространство. Рассмотрим пространства $L_{p}^{\text {loc }}(\mathbb{R} ; H)$ и $L_{\infty}^{\text {loc }}(\mathbb{R} ; H)$. Аналогично пространственному усреднению введем понятие усреднения по времени в локальной слабой или локальной *-слабой топологии этих пространств. Нам также потребуется понятие равномерной усредняемости относительно трансляций вдоль оси времени $\mathbb{R}$.

Рассмотрим некоторое семейство $\left\{f_{\varepsilon}(t), \varepsilon>0\right\}$ функций из $L_{p}^{\text {loc }}(\mathbb{R} ; H)$, и пусть $\widehat{f}(t) \in L_{p}^{\text {loc }}(\mathbb{R} ; H)$. По определению функция $\widehat{f}(t)$ является равномерным средним для $f_{\varepsilon}(t)$ при $\varepsilon \rightarrow 0+$ в $L_{p, w}^{\text {loc }}(\mathbb{R} ; H)$, если для любого $T>0$ и каждой функции $\varphi \in L_{q}(0, T ; H)$

$$
\int_{0}^{T}\left\langle f_{\varepsilon}(t+h), \varphi(t)\right\rangle d t \rightarrow \int_{0}^{T}\langle\widehat{f}(t+h), \varphi(t)\rangle d t \quad \text { при } \varepsilon \rightarrow 0+
$$

равномерно по $h \in \mathbb{R}$. Аналогично определяется равномерное среднее в локальной $*$-слабой топологии пространства $L_{\infty, * w}^{\text {loc }}(\mathbb{R} ; H)$. В этом случае предел $(1.14)$ должен иметь место для любой функции $\varphi \in L_{1}(0, M ; H)$.

Начнем рассмотрение с функций вида $g(t / \varepsilon)$, где $g(\tau) \in L_{p}^{\text {loc }}(\mathbb{R} ; H)$. Будем предполагать, что функция $g(t / \varepsilon)$ имеет равномерное среднее $\bar{g} \in H$. Сформулируем достаточное условие равномерной усредняемости к постоянной функции.

Теорема 1.5. Пусть $\left.\left.g(\tau) \in L_{p}^{b}(\mathbb{R} ; H), p \in\right] 1, \infty\right]$. Предположим, что

$$
\frac{1}{\lambda} \int_{h}^{h+\lambda} g(s) d s \rightarrow \bar{g} \quad(\lambda \rightarrow \infty)
$$

по норме пространства $H$ равномерно по $h \in \mathbb{R}$. Тогда функиия $g(t / \varepsilon)$ имеет равномерное среднее $\bar{g}$ в $L_{p, w}^{\mathrm{loc}}(\mathbb{R} ; H)$ (соответственно в $L_{\infty, * w}^{\mathrm{loc}}(\mathbb{R} ; H)$, если $p=\infty)$.

ДокАзАтЕльство. Аналогично пространственному случаю (см. теорему 1.1) достаточно проверить (1.14) только для ступенчатых функций вида

$$
\varphi(t)=\sum_{j=1}^{N} g_{j} \chi_{\left[T_{1 j}, T_{2 j}\right]}(t),
$$

где $g_{j} \in H$. Осталось заметить, что случай $\varphi=g \chi_{\left[T_{1}, T_{2}\right]}(t)$ прямо следует из (1.15). 
ПримеР 1.4. Пусть $g(t) \in C_{\boldsymbol{b}}(\mathbb{R} ; H)$ является почти периодической функцией по $t$ со значениями в $H$. Тогда, как известно (см. [5]), условие (1.15) выполнено для некоторой функции $\bar{g} \in H$, т.е. функция $g(t / \varepsilon)$ имеет равномерное среднее $\bar{g}$ в $L_{\infty, * w}^{\text {loc }}(\mathbb{R} ; H)$.

Введем понятие транслячионно-компактной (тр.-к.) функции в пространствах $C^{\text {loc }}(\mathbb{R} ; H)$ и $L_{p}^{\text {loc }}(\mathbb{R} ; H)$, которое расширяет понятие почти периодической функции.

ОПРЕДЕЛЕНИЕ 1.1. ФУнкция $g(t) \in C^{\mathrm{loc}}(\mathbb{R} ; H)$ называется трансляционнокомпактной в пространстве $C^{\mathrm{loc}}(\mathbb{R} ; H)$, если множество ее сдвигов $\{g(t+h)$ : $h \in \mathbb{R}\}$ предкомпактно в $C^{\text {loc }}(\mathbb{R} ; H)$, т.е. оно предкомпактно в $C([-M, M] ; H)$ для любого $M>0$.

Аналогично, функция $g(t) \in L_{p}^{\mathrm{loc}}(\mathbb{R} ; H)$ назьвается трансляционно-компактной в $L_{p}^{\text {loc }}(\mathbb{R} ; H)$, если множество ее сдвигов $\{g(t+h): h \in \mathbb{R}\}$ предкомпактно в пространстве $L_{p}(-M, M ; H)$ для любого $M>0$.

Множество $\mathscr{H}(g)=[\{g(t+h): h \in \mathbb{R}\}]_{\Xi}$ называется оболочкой функции $g$ пространстве $\Xi$. Здесь $[\cdot] \Xi$ обозначает замькание в пространстве $\Xi$, причем $\Xi=$ $C^{\text {loc }}(\mathbb{R} ; H)$, если $g$ тр.-к. в $C^{\text {loc }}(\mathbb{R} ; H) ; \Xi=L_{p}^{\text {loc }}(\mathbb{R} ; H)$, если $g$ тр.-к. в $L_{p}^{\text {loc }}(\mathbb{R} ; H)$. Отметим, что множество $\mathscr{H}(g)$ компактно в $\Xi$ и является трансляционно инвариантным, т.е. для любой функции $g_{1}(t) \in \mathscr{H}(g)$ функция $g_{1}(t+h) \in \mathscr{H}(g)$ для всякого $h \in \mathbb{R}$. (Подробнее о свойствах тр.-к. функций см. [6].)

$\mathrm{C}$ помощью тр.-к. функций можно построить новые примеры равномерно усредняемых функций.

Сформулируем сначала основные свойства равномерной усредняемости.

УТВЕРЖДЕНИЕ 1.1. Предположим, что функиия $g_{0}(\tau)$ является тр.-к. в пространстве $\left.L_{p}^{\mathrm{loc}}(\mathbb{R} ; H), p \in\right] 1, \infty\left[\right.$, а функиия $g_{0}(t / \varepsilon)$ имеет равномерное среднее $\bar{g}_{0} \in H$ в $L_{p, w}^{\text {loc }}(\mathbb{R} ; H)$. Тогда

(i) для каждой $\varphi \in L_{q}(0, T ; H), q=p /(p-1)$,

$$
\int_{0}^{T}\left\langle g\left(\frac{t}{\varepsilon}\right), \varphi(t)\right\rangle d t \rightarrow \int_{0}^{T}\left\langle\bar{g}_{0}, \varphi(t)\right\rangle d t \quad n p u \quad \varepsilon \rightarrow 0+
$$

равномерно по $g(\tau) \in \mathscr{H}\left(g_{0}(\tau)\right)$, әде $\mathscr{H}\left(g_{0}\right)$ является оболочкой функuи и $g_{0}(\tau)$

(ii) имеет место предел

$$
\mathscr{H}\left(g_{0}\left(\frac{t}{\varepsilon}\right)\right) \rightarrow\left\{\bar{g}_{0}\right\}(\varepsilon \rightarrow 0+)
$$

в топологии пространства $L_{p, w}^{\mathrm{loc}}(\mathbb{R} ; H)$, т.е. для любой окрестности $\mathscr{O}\left(\bar{g}_{0}\right)$ точки $\bar{g}_{0}$ в пространстве $L_{p, w}^{\text {loc }}(\mathbb{R} ; H)$ существует $\varepsilon_{0}>0$ такое, что $\mathscr{H}\left(g_{0}(t / \varepsilon)\right) \subseteq \mathscr{O}\left(\bar{g}_{0}\right)$ для любого $\varepsilon<\varepsilon_{0}$. 
ДокАЗАТЕЛЬСТво. Прежде всего заметим, что (ii) следует из (i), так как функции $\varphi \in L_{q}(0, T ; H)$ порождают базу топологии в пространстве $L_{p, w}^{\mathrm{loc}}(\mathbb{R} ; H)$. Докажем теперь (i). Достаточно проверить, что для каждой $\varphi \in H$

$$
\frac{1}{\lambda} \int_{0}^{\lambda}\langle g(\tau), \varphi\rangle d \tau \rightarrow\left\langle\bar{g}_{0}, \varphi\right\rangle \quad(\lambda \rightarrow \infty)
$$

равномерно по $g(\tau) \in \mathscr{H}\left(g_{0}(\tau)\right)$. Поскольку $\bar{g}_{0} \in H$ - равномерное среднее для $g(t / \varepsilon)$ в $L_{p, w}^{\text {loc }}(\mathbb{R} ; H)$, то для каждой $\varphi \in H$

$$
\frac{1}{\lambda} \int_{0}^{\lambda}\left\langle g_{0}(\tau+h), \varphi\right\rangle d \tau \rightarrow\left\langle\bar{g}_{0}, \varphi\right\rangle \quad(\lambda \rightarrow \infty)
$$

равномерно по $h \in \mathbb{R}$. Поскольку функция $g_{0}(\tau)$ является трансляционно-компактной в $L_{p}^{\text {loc }}(\mathbb{R} ; H)$, любая функция $g(\tau) \in \mathscr{H}\left(g_{0}\right)$ может быть приближена функциями вида $g_{0}\left(\tau+h_{i}\right)$ в сильной топологии пространства $L_{p}^{\text {loc }}(\mathbb{R} ; H)$. Следовательно, (1.19) влечет (1.18).

Теперь рассмотрим примеры, в которых функции вида $g(t, t / \varepsilon)$ имеют равномерные средние $\bar{g}(t)$, зависящие от времени $t$.

ПримеР 1.5. Пусть $\left.\left.g_{2}(\tau) \in L_{p}^{b}(\mathbb{R} ; H), p \in\right] 1, \infty\right]$. Предположим, что функция $g_{2}(t / \varepsilon)$ имеет равномерное среднее $\bar{g}_{2} \in H$ в $L_{p, w}^{\text {loc }}(\mathbb{R} ; H)$ и $g_{2}(\tau)$ - тр.-к. функция в $L_{p}^{\text {loc }}(\mathbb{R} ; H)$. Пусть также $g_{1}(t) \in C_{b}(\mathbb{R})$ и пусть $g_{1}(t)$ - тр.-к. в $C_{b}(\mathbb{R})$. Тогда функция $g(t, t / \varepsilon)=g_{1}(t) g_{2}(t / \varepsilon)$ имеет равномерное среднее $g_{1}(t) \bar{g}_{2}$ в $L_{p, w}^{\text {loc }}(\mathbb{R} ; H)$ при $\varepsilon \rightarrow 0+$.

Докажем это утверждение. Зафиксируем любой замкнутый интервал $[0, T]$ и произвольное число $\varepsilon>0$. Поскольку множество $\left\{g_{1}(t+h), h \in \mathbb{R}\right\}$ предкомпактно в $C([0, T] ; H)$, можно выбрать его $\varepsilon$-сеть $\left\{g_{1}\left(t+h_{i}\right), i=1, \ldots, N\right\}$. Тогда для любого $h \in \mathbb{R}$ сушествует $i \in\{1, \ldots, N\}$ такое, что

$$
\max _{t \in[0, T]}\left|g_{1}(t+h)-g_{1}\left(t+h_{i}\right)\right|<\varepsilon
$$

Функция $g_{2}(t / \varepsilon)$ имеет равномерное среднее в $L_{p, w}^{\text {loc }}(\mathbb{R} ; H)$. Следовательно, для любой функции $\varphi \in L_{q}(0, T ; H)$ и любого $i=1, \ldots, N$

$$
\int_{0}^{T}\left\langle g_{2}\left(\frac{t+h}{\varepsilon}\right), g_{1}\left(t+h_{i}\right) \varphi(t)\right\rangle d t \rightarrow \int_{0}^{T}\left\langle\bar{g}_{2}, g_{1}\left(t+h_{i}\right) \varphi(t)\right\rangle d t \text { при } \varepsilon \rightarrow 0+
$$

равномерно по $h \in \mathbb{R}$. Тем самым,

$$
\begin{aligned}
\int_{0}^{T}\left\langle g_{1}(t+h)\left(g_{2}\left(\frac{t+h}{\varepsilon}\right)-\bar{g}_{2}\right), \varphi(t)\right\rangle d t \\
=\int_{0}^{T}\left\langle\left(g_{2}\left(\frac{t+h}{\varepsilon}\right)-\bar{g}_{2}\right), g_{1}\left(t+h_{i}\right) \varphi(t)\right\rangle d t \\
\quad+\int_{0}^{T}\left\langle\left(g_{2}\left(\frac{t+h}{\varepsilon}\right)-\bar{g}_{2}\right),\left(g_{1}(t+h)-g_{1}\left(t+h_{i}\right)\right) \varphi(t)\right\rangle d t
\end{aligned}
$$


Поскольку $g_{2}(\tau) \in L_{p}^{b}(\mathbb{R} ; H)$, мы можем, используя (1.20), оценить интеграл (1.24) числом

$$
C \max _{t \in[0, T]}\left|g_{1}(t+h)-g_{1}\left(t+h_{i}\right)\right|<C \varepsilon \quad(\text { где } i \text { зависит от } h) .
$$

В силу (1.21) интеграл (1.23) стремится к нулю при $\varepsilon \rightarrow 0+$ равномерно по $h \in \mathbb{R}$. Поэтому интеграл (1.22) стремится к нулю при $\varepsilon \rightarrow 0+$ равномерно по $h \in \mathbb{R}$. Мы доказали, что

$$
\int_{0}^{T}\left\langle g_{1}(t+h) g_{2}\left(\frac{t+h}{\varepsilon}\right), \varphi(t)\right\rangle d t \rightarrow \int_{0}^{T}\left\langle g_{1}(t+h) \bar{g}_{2}, \varphi(t)\right\rangle d t \quad \text { при } \varepsilon \rightarrow 0+
$$

равномерно по $h \in \mathbb{R}$. Следовательно, $g_{1}(t) \bar{g}_{2}$ является равномерным средним для функции $g_{1}(t) g_{2}(t / \varepsilon)$.

Из соотношения (1.25) следует, что для функции $g(t, t / \varepsilon)=g_{1}(t) g_{2}(t / \varepsilon)$ выполнено свойство, аналогичное свойству (ii) из утверждения 1.1. Ясно, что функции $g_{1}(t) g_{2}(t / \varepsilon)$ и $g_{1}(t) \bar{g}_{2}$ являются тр.-к. в $L_{p}^{\text {loc }}(\mathbb{R} ; H)$. Рассмотрим их оболочки $\mathscr{H}\left(g_{1}(t) g_{2}(t / \varepsilon)\right)$ и $\mathscr{H}\left(g_{1}(t) \bar{g}_{2}\right)$ в $L_{p}^{\text {loc }}(\mathbb{R} ; H)$.

УТВЕРЖДЕНИЕ 1.2. В топологии пространства $L_{p, w}^{\text {loc }}(\mathbb{R} ; H)$ въцполнено предельное соотношение

$$
\mathscr{H}\left(g_{1}(t) g_{2}\left(\frac{t}{\varepsilon}\right)\right) \rightarrow \mathscr{H}\left(g_{1}(t) \bar{g}_{2}\right) \quad(\varepsilon \rightarrow 0+)
$$

Доказательство аналогично доказательству утверждения 1.1.

ПримеР 1.6. Пусть $g_{1} \in C_{b}(\mathbb{R} ; \mathbb{R})$ и $g_{2} \in C_{b}(\mathbb{R} ; H)$. Кроме того, предположим, что функции $g_{1}$ и $g_{2}$ - почти периодические в этих пространствах. Тогда функция $g(t, t / \varepsilon)=g_{1}(t) g_{2}(t / \varepsilon)$ удовлетворяет всем условиям из примера 1.5 и, следовательно, имеет среднее в пространстве $L_{\infty, * w}^{\text {loc }}(\mathbb{R} ; H)$. В частности, если $\bar{g}_{2} \in H-$ среднее для $g_{2}$, то $\mathscr{H}\left(g_{1}(t) g_{2}(t / \varepsilon)\right) \rightarrow \mathscr{H}\left(g_{1}(t) \bar{g}_{2}\right) \quad(\varepsilon \rightarrow 0+)$ *-слабо в $L_{\infty, * w}^{\mathrm{loc}}(\mathbb{R} ; H)$.

Тем же способом доказывается, что функция вида

$$
g_{N}\left(t, \frac{t}{\varepsilon}\right)=\sum_{i=1}^{N} g_{1 i}(t) g_{2 i}\left(\frac{t}{\varepsilon}\right)+\sum_{i=1}^{N} g_{3 i}\left(\frac{t}{\varepsilon}\right) g_{4 i}(t)
$$

имеет равномерное среднее

$$
\bar{g}_{N}(t)=\sum_{i=1}^{N} g_{1 i}(t) \bar{g}_{2 i}+\sum_{i=1}^{N} \bar{g}_{3 i} g_{4 i}(t)
$$

если известно, что функции $g_{1 i}, g_{3 i} \in C_{b}(\mathbb{R} ; \mathbb{R}), g_{2} i, g_{4 i} \in C_{b}(\mathbb{R} ; H)$ и они являются почти периодическими в этих пространствах. Аналогичные результаты имеют место для предела функций $g_{N}(t, t / \varepsilon)$ вида (1.27) при $N \rightarrow \infty$ в соответствуюшем функциональном пространстве. 


\section{§2. Траекторные аттракторы эволюционных уравнений}

В этом параграфе излагается обшая схема построения траекторных аттракторов эволюционных уравнений. В следуюшем параграфе эта схема применяется для изучения траекторных аттракторов конкретных уравнений с быстро осциллирующими коэффициентами и соответствующих им усредненных уравнений.

Рассматривается абстрактное автономное эволюционное уравнение

$$
\partial_{t} u=A(u), \quad t \geqslant 0
$$

Предполагается заданным (нелинейный) оператор $A(\cdot): E \rightarrow E_{0}$, где $E, E_{0}$ - банаховы пространства и $E \subseteq E_{0}$. Например, $A(u)=a \Delta u-f(u)+g($ см. $\S 3)$.

Мы будем исследовать решения $u(s)$ уравнения $(2.1)$ в целом, как функции переменной $s \in \mathbb{R}_{+}$. Здесь $s \equiv t$ обозначает переменную времени. Множество решений (2.1) будет называться пространством траекторий $\mathscr{K}^{+}$уравнения (2.1). Опишем пространство траекторий $\mathscr{K}^{+}$более подробно.

Прежде всего рассмотрим решения $u(s)$ уравнения $(2.1)$, определенные на фиксированном отрезке времени $\left[t_{1}, t_{2}\right] \subset \mathbb{R}_{+}$. Изучаются решения уравнения $(2.1)$, принадлежащие банахову пространству $\mathscr{F}_{t_{1}, t_{2}}$. Пространство $\mathscr{F} t_{1}, t_{2}$ состоит из функций $f(s), s \in\left[t_{1}, t_{2}\right]$, таких, что $f(s) \in E$ при почти всех $s \in\left[t_{1}, t_{2}\right]$. Например, $\mathscr{F}_{t_{1}, t_{2}}$ может быть или пространством $C\left(\left[t_{1}, t_{2}\right] ; E\right)$, или $L_{p}\left(t_{1}, t_{2} ; E\right), p \in$ $[1, \infty]$, или пересечением подобных пространств (см. 33 ). Предполагается, что $\Pi_{t_{1}, t_{2}} \mathscr{F}_{t_{3}, t_{4}}=\mathscr{F}_{t_{1}, t_{2}}$ при $\left[t_{1}, t_{2}\right] \subset\left[t_{3}, t_{4}\right]$, где $\Pi_{t_{1}, t_{2}}$ обозначает оператор сужения на отрезок $\left[t_{1}, t_{2}\right]$.

Предполагается, что если $f(s) \in \mathscr{F}_{t_{1}, t_{2}}$, то $A(f(s)) \in \mathscr{D}_{t_{1}, t_{2}}$, где $\mathscr{D}_{t_{1}, t_{2}}$ - некоторое более широкое чем $\mathscr{F}_{t_{1}, t_{2}}$ банахово пространство, $\mathscr{F}_{t_{1}, t_{2}} \subseteq \mathscr{D}_{t_{1}, t_{2}}$. Производная $\partial_{t} f(t)$ - обобщенная функция со значениями в $E_{0}, \partial_{t} f(s) \in D^{\prime}(] t_{1}, t_{2}\left[; E_{0}\right)$,

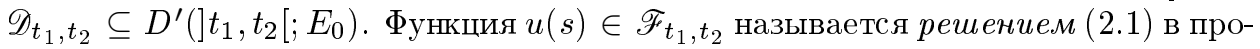
странстве $\mathscr{F}_{t_{1}, t_{2}}$ (на интервале $] t_{1}, t_{2}\left[\right.$ ), если $\partial_{t} u(s)=A(u(s))$ в смысле обобщенных функций.

Определим также пространство

$$
\mathscr{F}_{+}^{\text {loc }}=\left\{f(s), s \in \mathbb{R}_{+}: \Pi_{t_{1}, t_{2}} f(s) \in \mathscr{F}_{t_{1}, t_{2}} \quad \forall\left[t_{1}, t_{2}\right] \subseteq \mathbb{R}_{+}\right\}
$$

Например, если $\mathscr{F}_{t_{1}, t_{2}}=C\left(\left[t_{1}, t_{2}\right] ; E\right)$, то $\mathscr{F}_{+}^{\text {loc }}=C^{\text {loc }}\left(\mathbb{R}_{+} ; E\right)$, а если $\mathscr{F}_{t_{1}, t_{2}}=$ $L_{p}\left(t_{1}, t_{2} ; E\right)$, то $\mathscr{F}_{+}^{\text {loc }}=L_{p}^{\text {loc }}\left(\mathbb{R}_{+} ; E\right)$.

Функция $u(s) \in \mathscr{F}_{+}^{\text {loc }}$ называется решением уравнения $(2.1)$ в $\mathscr{F}_{+}^{\text {loc }}$, если $\Pi_{t_{1}, t_{2}} u(s)$ является решением $(2.1)$ в $\mathscr{F}_{t_{1}, t_{2}}$ для любого $\left[t_{1}, t_{2}\right] \subset \mathbb{R}_{+}$.

Через $\mathscr{K}^{+}$обозначается некоторое множество решений $(2.1)$, принадлежащих $\mathscr{F}_{+}^{\text {loc }}$. (Заметим, что $\mathscr{K}^{+}$не обязательно является множеством всех решений $(2.1)$, принадлежащих $\mathscr{F}_{+}^{\text {loc }}$.) Элементы множества $\mathscr{K}^{+}$будут называться траекториями, а само множество $\mathscr{K}^{+}$- пространством траекторий уравнения (2.1).

Предполагается, что пространство траекторий $\mathscr{K}^{+}$является трансляиионно-инвариантным в следуюшем смысле: если $u(s) \in \mathscr{K}^{+}$, то $u(h+s) \in \mathscr{K}^{+}$ для любого $h \geqslant 0$. Это условие является естественным свойством решений автономного уравнения.

Рассмотрим теперь операторы сдвигов $T(h)$ в $\mathscr{F}_{+}^{\text {loc }}$ :

$$
T(h) f(s)=f(s+h) \text { для } h \geqslant 0 .
$$


Ясно, что множество отображений $\{T(h), h \geqslant 0\}$ образует полугруппу в $\mathscr{F}_{+}^{\text {loc }}$ : $T\left(h_{1}\right) T\left(h_{2}\right)=T\left(h_{1}+h_{2}\right)$ при $h_{1}, h_{2} \geqslant 0$ и $T(0)=I$. Заменим переменную $h$ на переменную времени $t$. Полугруппа $\{T(t), t \geqslant 0\}$ назьвается полугруппой сдвигов. В силу сделанного предположения полугруппа сдвигов отображает пространство траекторий $\mathscr{K}^{+}$на себя:

$$
T(t) \mathscr{K}^{+} \subseteq \mathscr{K}^{+} \text {при всех } t \geqslant 0
$$

Ниже изучаются свойства притяжения полугруппы сдвигов $\{T(t)\}$, действующей на пространстве траекторий $\mathscr{K}^{+} \subset \mathscr{F}_{+}^{\text {loc }}$. Определим топологию в пространстве $\mathscr{F}_{+}^{\text {loc }}$.

Пусть задана некоторая топология сходимости в $\mathscr{F}_{t_{1}, t_{2}}$. Обозначим через $\Theta_{t_{1}, t_{2}}$ топологическое пространство $\mathscr{F}_{t_{1}, t_{2}}$, снабженное этой топологией. Предполагается, что $\Theta_{t_{1}, t_{2}}$ - хаусдорфово пространство. Например, $\Theta_{t_{1}, t_{2}}$ может быть само $\mathscr{F}_{t_{1}, t_{2}}$ с топологией сильной или слабой (или даже $*$-слабой) сходимости этого банахова пространства. Обозначим через $\Theta_{+}^{\text {loc }}$ пространство $\mathscr{F}_{+}^{\text {loc }}$, снабженное топологией локальной сходимости на $\Theta_{t_{1}, t_{2}}$ при любом $\left[t_{1}, t_{2}\right] \subseteq \mathbb{R}_{+}$. Точнее, по определению последовательность функций $\left\{f_{n}(s)\right\} \subset \mathscr{F}_{+}^{\text {loc }}$ сходится к функции $f(s) \in \mathscr{F}_{+}^{\text {loc }}$ при $n \rightarrow \infty$ в $\Theta_{+}^{\text {loc }}$, если $\Pi_{t_{1}, t_{2}} f_{n}(s) \rightarrow \Pi_{t_{1}, t_{2}} f(s)(n \rightarrow \infty)$ в $\Theta_{t_{1}, t_{2}}$ для любого $\left[t_{1}, t_{2}\right] \subseteq \mathbb{R}_{+}$. Нетрудно доказать, что пространство $\Theta_{+}^{\text {loc }}$ является пространством Хаусдорфа. Заметим, что полугруппа сдвигов $\{T(t)\}$ непрерывна в $\Theta_{+}^{\text {loc }}$. Это утверждение непосредственно вытекает из определения топологического пространства $\Theta_{+}^{\text {loc }}$.

Определим также следующее банахово пространство:

$$
\mathscr{F}_{+}^{b}:=\left\{f(s) \in \mathscr{F}_{+}^{\text {loc }}:\|f\|_{\mathscr{F}_{+}^{b}}<+\infty\right\},
$$

где норма

$$
\|f\|_{\mathscr{F}_{+}^{b}}=\sup _{t \geqslant 0}\left\|\Pi_{0,1} f(t+s)\right\|_{\mathscr{F}_{0,1}} .
$$

Отметим, что $\mathscr{F}_{+}^{b} \subseteq \Theta_{+}^{\text {loc }}$. Пространство $\mathscr{F}_{+}^{b}$ необходимо для задания ограниченных множеств в $\mathscr{K}^{+}$.

Будем предполагать, что $\mathscr{K}^{+} \subseteq \mathscr{F}_{+}^{b}$, т.е. любая траектория $u(s) \in \mathscr{K}^{+}$уравнения (2.1) имеет конечную норму (2.4). Приведем определение траекторного аттрактора полугрупшы сдвигов $\{T(t)\}$, действующей на $\mathscr{K}^{+}$.

ОПРЕДЕЛЕНИЕ 2.1. Множество $P \subseteq \Theta_{+}^{\text {loc }}$ называется притягивающим для пространства траекторий $\mathscr{K}^{+}$в топологии $\Theta_{+}^{\text {loc }}$, если для любого ограниченного в $\mathscr{F}_{+}^{b}$ множества $B \subseteq \mathscr{K}^{+}$множество $P$ притягивает $T(t) B$ при $t \rightarrow+\infty$ в топологии $\Theta_{+}^{\text {loc }}$, т.е. для любой окрестности $\mathscr{O}(P)$ в $\Theta_{+}^{\text {loc }}$ существует $t_{1} \geqslant 0$ такое, что $T(t) B \subseteq \mathscr{O}(P)$ при любом $t \geqslant t_{1}$.

ОПРЕДЕЛЕНИЕ 2.2. Множество $\mathfrak{A} \subseteq \mathscr{K}^{+}$назьвается траекторным аттрактором полугрупшы сдвигов $\{T(t)\}$ на $\mathscr{K}^{+}$в топологии $\Theta_{+}^{\text {loc }}$, если

(i) $\mathfrak{A}$ компактно в $\Theta_{+}^{\text {loc }}$;

(ii) множество $\mathfrak{A}$ строго инвариантно относительно полугрупшы сдвигов: $T(t) \mathfrak{A}=\mathfrak{A}$ при всех $t \geqslant 0 ;$

(iii) $\mathfrak{A}$ - притягивающее множество для $\mathscr{K}^{+}$. 
ЗАмечАнИЕ 2.1. Используя терминологию из [7], можно сказать, что траекторный аттрактор $\mathfrak{A}$ является глобальным $\left(\mathscr{F}_{+}^{b}, \Theta_{+}^{\text {loc }}\right)$-аттрактором полугруппы сдвигов $\{T(t)\}$, действуюшей на $\mathscr{K}^{+}$, т.е. $\mathfrak{A}$ притягивает $T(t) B$ при $t \rightarrow+\infty$ в топологии $\Theta_{+}^{\text {loc }}$, где $B$ - любое ограниченное в $\mathscr{F}_{+}^{b}$ множество.

Сформулируем основную теорему о существовании траекторного аттрактора уравнения (2.1).

TEOРема 2.1. Пусть пространство траекторий $\mathscr{K}^{+}, \mathscr{K}^{+} \subseteq \mathscr{F}_{+}^{b}$, соответствующее уравнению $(2.1)$, замкнуто в $\Theta_{+}^{\text {loc }}$. Предполагается, что существует притягивающее множество $P$ для $\{T(t)\}$ на $\mathscr{K}^{+}$в топологии пространства $\Theta_{+}^{\mathrm{loc}}$ такое, что $P$ компактно в $\Theta_{+}^{\mathrm{loc}}$ и ограничено в $\mathscr{F}_{+}^{b}$. Тогда полугруппа сдвигов $\{T(t), t \geqslant 0\}$, действующая на $\mathscr{K}^{+}$, имеет траекторный аттрактор $\mathfrak{A} \subseteq \mathscr{K}^{+} \cap P$. Множество $\mathfrak{A}$ компактно в $\Theta_{+}^{\text {loc }}$ и ограничено в $\mathscr{F}_{+}^{b}$.

ДоказАтельство. В самом деле, полугруппа $\{T(t)\}$ непрерывна на $\mathscr{K}^{+}$в топологии $\Theta_{+}^{\text {loc }}$. Множество $P$ является $\left(\mathscr{F}_{+}^{b}, \Theta_{+}^{\text {loc }}\right)$-притягивающим, компактным в $\Theta_{+}^{\text {loc }}$ и ограниченным в $\mathscr{F}_{+}^{b}$. Тогда полугруппа $\{T(t)\}$ имеет глобальный $\left(\mathscr{F}_{+}^{b}, \Theta_{+}^{\text {loc }}\right)$-аттрактор $\mathfrak{A}$, который, очевидно, является также траекторным аттрактором (см. полное доказательство в [6]).

Опишем структуру траекторного аттрактора $\mathfrak{A}$ уравнения (2.1) в терминах полных траекторий этого уравнения.

Рассмотрим уравнение (2.1) на всей числовой оси времени:

$$
\partial_{t} u=A(u), \quad t \in \mathbb{R}
$$

Мы уже определили пространство траекторий $\mathscr{K}^{+}$уравнения $(2.5)$ на $\mathbb{R}_{+}$. Теперь распространим это определение на всю ось $\mathbb{R}$. Если функция $f(s), s \in \mathbb{R}$, задана на всей оси времени, то сдвиги $T(h) f(s)=f(s+h)$ также определены при отрицательных $h$.

Функция $u(s), s \in \mathbb{R}$, назьвается полной траекторией уравнения (2.5), если $\Pi_{+} u(s+h) \in \mathscr{K}^{+}$при любом $h \in \mathbb{R}$. Здесь $\Pi_{+}=\Pi_{0, \infty}$ обозначает оператор ограничения на полуось $\mathbb{R}_{+}$.

Мы ввели пространства $\mathscr{F}_{+}^{\text {loc }}, \mathscr{F}_{+}^{b}$ и $\Theta_{+}^{\text {loc }}$. Аналогичньм образом определяются пространства $\mathscr{F}^{\text {loc }}, \mathscr{F}^{b}$ :

$$
\begin{gathered}
\mathscr{F}^{\mathrm{loc}}=\left\{f(s), s \in \mathbb{R}: \Pi_{t_{1}, t_{2}} f(s) \in \mathscr{F}_{t_{1}, t_{2}} \forall\left[t_{1}, t_{2}\right] \subseteq \mathbb{R}\right\} ; \\
\mathscr{F}^{b}=\left\{f(s) \in \mathscr{F}^{\mathrm{loc}}:\|f\|_{\mathscr{F}^{b}}<+\infty\right\},
\end{gathered}
$$

где

$$
\|f\|_{\mathscr{F} b}=\sup _{h \in \mathbb{R}}\left\|\Pi_{0,1} f(h+s)\right\|_{\mathscr{F}_{0,1}}
$$

Топологическое пространство $\Theta^{\text {loc }}$ совпадает (как множество) с $\mathscr{F}$ loc , и по определению $f_{n}(s) \rightarrow f(s)(n \rightarrow \infty)$ в $\Theta^{\text {loc }}$, если $\Pi_{t_{1}, t_{2}} f_{n}(s) \rightarrow \Pi_{t_{1}, t_{2}} f(s)(n \rightarrow \infty)$ в $\Theta_{t_{1}, t_{2}}$ при любом $\left[t_{1}, t_{2}\right] \subseteq \mathbb{R}$. 
ОПредЕЛЕНИЕ 2.3. $Я \partial р о ~ \mathscr{K}$ в пространстве $\mathscr{F}^{b}$ уравнения (2.5) есть объединение всех полных траекторий $u(s), s \in \mathbb{R}$, уравнения $(2.5)$, ограниченных в $\mathscr{F}^{b}$ по норме (2.6):

$$
\left\|\Pi_{0,1} u(h+s)\right\|_{\mathscr{F}_{0,1}} \leqslant C_{u} \quad \forall h \in \mathbb{R} .
$$

ТЕОРема 2.2. Пусть выполненъ условия теоремы 2.1. Тогда

$$
\mathfrak{A}=\Pi_{+} \mathscr{K} .
$$

Множество $\mathscr{K}$ компактно в $\Theta^{\mathrm{loc}}$ и ограничено в $\mathscr{F}^{b}$.

Доказательство приведено в [6].

Поясним подробнее характер притяжения любого ограниченного множества $B$ из $\mathscr{K}^{+}$к траекторному аттрактору $\mathfrak{A}$.

СЛЕДСТВИЕ 2.1. Пусть выполнены условия теоремы 2.1. Пусть В - ограниченное в $\mathscr{F}_{+}^{b}$ множество из $\mathscr{K}^{+}$. Тогда при любом $M>0$ множсество $\Pi_{0, M} T(t) B$ стремится $\kappa \Pi_{0, M} \mathscr{K}$ в топологическом пространстве $\Theta_{0, M}$ при $t \rightarrow \infty$. Например, если $\Theta_{0, M}-$ метризуемое пространство, то

$\operatorname{dist}_{\Theta_{0, M}}\left(\Pi_{0, M} T(t) B, \Pi_{0, M} \mathfrak{A}\right)=\operatorname{dist}_{\Theta_{0, M}}\left(\Pi_{0, M} T(t) B, \Pi_{0, M} \mathscr{K}\right) \rightarrow 0 \quad(t \rightarrow \infty)$.

Здесь расстояние от множества $X$ до множества $Y$ в метрическом пространстве $\mathscr{M}$ определяется по Хаусдорфу: $\operatorname{dist} \mathscr{M}(X, Y)=\sup _{x \in X} \inf _{y \in Y} \rho_{\mathscr{M}}(x, y)$, где $\rho_{\mathscr{M}}(x, y)$ обозначает метрику в $\mathscr{M}$.

Теоремы 2.1 и 2.2 показывают, что для построения траекторного аттрактора необходимо установить существование притягивающего множества $P$, компактного в $\Theta_{+}^{\text {loc }}$ и ограниченного в $\mathscr{F}_{+}^{b}$. Обычно в приложениях таким притягивающим множеством служит шар большого радиуса $B_{R}=\left\{\|f\|_{\mathscr{F}_{+}^{b}} \leqslant R\right\}$ в $\mathscr{F}_{+}^{b}(R \gg 1)$. Сушествование такого множества $B_{R}$ обычно следует из неравенства вида

$$
\|T(t) u\|_{\mathscr{F}_{+}^{b}} \leqslant C\left(\|u\|_{\mathscr{F}_{+}^{b}}\right) e^{-\gamma t}+R_{0}, \quad \gamma>0
$$

при любом $u \in \mathscr{K}^{+}$и всех $t \geqslant 0$, где $C(R)$ зависит от $R$, а $R_{0}$ не зависит от $u$. Как правило, неравенство (2.9) вытекает из априорных оценок для решений уравнения (2.1).

Теперь мы коротко опишем построение траекторных аттракторов для неавтономных эволюционных уравнений. Неавтономные уравнения содержат члены, явно зависящие от времени. Например, ими могут быть внешние силы, функции взаимодействия или иные коэффициенты, входящие в уравнение. Поэтому нам удобно ввести функцию $\sigma(t), t \in \mathbb{R}$, называемую временныોм символом рассматриваемого уравнения, которая состоит из всех членов изучаемого уравнения, зависящих от времени. Значения функции $\sigma(t)$ принадлежат соответствуюшему функциональному пространству.

Неавтономное уравнение можно записать в виде

$$
\partial_{t} u=A_{\sigma(t)}(u), \quad t \geqslant 0 .
$$

Здесь, подобно уравнению (2.1), введен оператор $A_{\sigma(s)}(\cdot): E \rightarrow E_{0}, s \in \mathbb{R}$. Например, $A_{\sigma(t)}(u)=a \Delta u-f(u)+g(x, t)$, где $\sigma(t)=g(x, t)$. 
Временно̀ й символ $\sigma(s)$ как функция времени принадлежит некоторому топо-

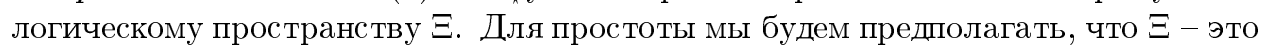
пространство $C^{\text {loc }}(\mathbb{R} ; H)$ или $\Xi=L_{p}^{\text {loc }}(\mathbb{R} ; H)$, снабженное соответствующей топологией локальной сходимости на любом конечном отрезке числовой оси (см. п. 1.2). При построении траекторного аттрактора уравнения (2.10) мы исходим из следующего требования: аттрактор не должен меняться при замене символа $\sigma(s)$ любым сдвинутым символом $\sigma(s+h), h \in \mathbb{R}$. Поэтому мы будем рассматривать целое семейство уравнений $(2.10)$ с различными символами $\sigma(s) \in \Sigma$, где $\Sigma$ является трансляционно-инвариантным множеством:

$$
T(h) \Sigma=\Sigma \quad \forall h \in \mathbb{R} .
$$

Обычно в приложениях $\Sigma$ совпадает с оболочкой $\mathscr{H}\left(\sigma_{0}\right)$ (в $\Xi$ ) некоторого начального символа $\sigma_{0}(s)$ изучаемого уравнения:

$$
\Sigma=\mathscr{H}\left(\sigma_{0}\right)=\left[\left\{\sigma_{0}(t+h): h \in \mathbb{R}\right\}\right] \Xi .
$$

Мы будем также предполагать, что функция $\sigma_{0}(s)$ является трансляционно-компактной в пространстве $\Xi$ (см. п. 1.2). Следовательно, множество $\Sigma$ компактно в $\Xi$. Например, функция $\sigma_{0}(t)$ может быть периодической, квазипериодической или почти периодической. Тогда ее оболочка $\mathscr{H}\left(\sigma_{0}\right)$ компактна в пространстве $C_{b}(\mathbb{R} ; H)$, и, тем самьм, множество $\Sigma=\mathscr{H}\left(\sigma_{0}\right)$ компактно в $\Xi=C^{\text {loc }}(\mathbb{R} ; H)$.

Как и в автономном случае, будем предполагать, что каждому символу $\sigma \in \Sigma$ соответствует пространство траекторий $\mathscr{K}_{\sigma}^{+} \subset \mathscr{F}_{+}^{b}$, состоящее из решений $u(s)$, $s \geqslant 0$, уравнения (2.10). Функция $u(t)$ удовлетворяет уравнению в смысле обобщенных функций пространства $D^{\prime}(] t_{1}, t_{2}\left[; E_{0}\right)$ при любом $\left[t_{1}, t_{2}\right] \subset \mathbb{R}_{+}$. Пространства $\mathscr{F}_{+}^{\text {loc }}, \mathscr{F}_{+}^{b}$ и $\Theta_{+}^{\text {loc }}$ имеют тот же смысл, что и в автономном случае.

Рассмотрим действие полугрупшы сдвигов $\{T(t)\}$ на $\mathscr{K}_{\sigma}^{+}$. Легко видеть, что в общем случае пространство траекторий $\mathscr{K}_{\sigma}^{+}$не инвариантно относительно $\{T(t)\}$, т.е. $T(t) \mathscr{K}_{\sigma}^{+} \nsubseteq \mathscr{K}_{\sigma}^{+}$. Однако выполнено включение:

$$
T(t) \mathscr{K}_{\sigma}^{+} \subseteq \mathscr{K}_{T(t) \sigma}^{+} \quad \forall t \geqslant 0 .
$$

Рассмотрим объединенное пространство траекторий

$$
\mathscr{K}_{\Sigma}^{+}=\bigcup_{\sigma \in \Sigma} \mathscr{K}_{\sigma}^{+},
$$

которое уже инвариантно относительно $\{T(t)\}$ :

$$
T(t) \mathscr{K}_{\Sigma}^{+} \subseteq \mathscr{K}_{\Sigma}^{+} \quad \forall t \geqslant 0 .
$$

Аналогично автономному случаю вводятся определения равномерного (по $\sigma \in$ $\Sigma)$ aтmрактора полугруппы сдвигов на $\mathscr{K}_{\Sigma}^{+}$в топологии $\Theta_{+}^{\text {loc }}$. По определению глобальный $\left(\mathscr{F}_{+}^{b}, \Theta_{+}^{\text {loc }}\right)$-аттрактор полугруппы сдвигов $\{T(t)\}$, действующей на $\mathscr{K}_{\Sigma}^{+}$, называется равномерным (по $\left.\sigma \in \Sigma\right)$ траекторным аттрактором $\mathfrak{A}_{\Sigma}$ уравнения $(2.10)$, т.е. множество $\mathfrak{A}_{\Sigma}$ компактно в $\Theta_{+}^{\text {loc }}$, притягивает $T(t) B$ при $t \rightarrow+\infty$ в топологии $\Theta_{+}^{\text {loc }}$ для любого ограниченного (в $\left.\mathscr{F}_{+}^{b}\right)$ множества $B$ из $\mathscr{K}_{\Sigma}^{+}$ и $T(t) \mathfrak{A}_{\Sigma}=\mathfrak{A}_{\Sigma}$ при $t \geqslant 0$.

По аналогии с автономньм случаем вводится также понятие ядра $\mathscr{K}_{\sigma}$ уравнения (2.10), которое состоит из всех траекторий $u(s), s \in \mathbb{R}$, уравнения (2.10), определенных на всей оси и ограниченных по норме $\mathscr{F}^{b}$ (см. (2.6)). 
ТЕОРема 2.3. Пусть пространство символов $\Sigma=\mathscr{H}\left(\sigma_{0}\right)$, где $\sigma_{0}(s)$ трансляционно-компактная функция в . Предположим, что обтединенное пространство траекторий $\mathscr{K}_{\Sigma}^{+}$, соответствующее уравнению (2.1), замкнуто в $\Theta_{+}^{\text {loc }}$ и $\mathscr{K}_{\Sigma}^{+} \subseteq \mathscr{F}_{+}^{b}$. Предположим также, что существует равномерно $($ по $\sigma \in \Sigma)$ притягивающее множсество $P$ для $\{T(t)\}$ на $\mathscr{K}_{\Sigma}^{+}$в топологии пространства $\Theta_{+}^{\text {loc }}$ такое, что $P$ компактно в $\Theta_{+}^{\text {loc }}$ и ограничено в $\mathscr{F}_{+}^{b}$. Тогда полугруппа сдвигов $\{T(t), t \geqslant 0\}$, действующая на $\mathscr{K}_{\Sigma}^{+}$, имеет равномерныи (по $\sigma \in \Sigma)$ траекторный аттрактор $\mathfrak{A}_{\Sigma} \subseteq \mathscr{K}^{+} \cap P$. При этом имеет место равенство:

$$
\mathfrak{A}_{\Sigma}=\Pi_{+} \bigcup_{\sigma \in \mathscr{H}\left(\sigma_{0}\right)} \mathscr{K}_{\sigma} .
$$

Множество $\mathscr{K}_{\sigma}$ не пусто при любом $\sigma \in \Sigma$, компактно в $\Theta_{+}^{\text {loc }}$ и ограничено в $\mathscr{F}_{+}^{b}$.

Доказательство теоремы 2.3 для более общих пространств символов $\Sigma$ изложено в [6].

В заключение мы установим связь между траекторными и глобальньми аттракторами. Рассмотрим автономное уравнение (2.1) и соответствующее пространство траекторий $\mathscr{K}^{+}$. Предположим, что при любом $u_{0} \in E$ сушествует и притом единственная траектория $u(s) \in \mathscr{K}^{+}$такая, что

$$
u(0)=u_{0}
$$

Тогда задача Коши (2.1), (2.14) порождает полугруппу $\{S(t), t \geqslant 0\}$, действуюшую в банаховом пространстве $E$ по формуле:

$$
S(t) u_{0}=u(t)
$$

Множество $\mathscr{A} \Subset E$ называется глобальным аттрактором полугруппы $\{S(t)\}$, если $S(t) \mathscr{A}=\mathscr{A}$ при всех $t \geqslant 0$ и для любого ограниченного в $E$ множества $B$

$$
\operatorname{dist}_{E}(S(t) B, \mathscr{A}) \rightarrow 0 \text { при } t \rightarrow \infty .
$$

Изучению глобальных аттракторов эволюционных уравнений при условии единственности решений задачи Коши посвяшена обширная литература (см., например, [7]-[9]). Приведем один результат, позволяющий сводить изучение глобального аттрактора к исследованию траекторного аттрактора. Рассмотрим пространство $C^{\text {loc }}\left(\mathbb{R}_{+} ; E\right)$, снабженное топологией локальной равномерной сходимости на любом интервале $\left[t_{1}, t_{2}\right] \subset \mathbb{R}_{+}$.

ТЕОРема 2.4. Пусть выполнены условия теоремы 2.1. Предположим, что задача Коши (2.1), (2.14) имеет единственное решение. Предположим также, что справедливо следующее вложение:

$$
\Theta_{+}^{\text {loc }} \subset C^{\text {loc }}\left(\mathbb{R}_{+} ; E\right) \text {. }
$$

Тогда полугруппа $\{S(t)\}$, отвечающая задаче (2.1), (2.14), имеет глобальньи аттрактор $\mathscr{A}$, причем

$$
\mathscr{A}=\mathfrak{A}(0)=\mathscr{K}(0)=\{u(0): u \in \mathscr{K}\},
$$

где $\mathfrak{A}$ и $\mathscr{K}$ - траекторный аттрактор и ядро уравнения (2.1). 
Доказательство очевидно вытекает из теорем 2.1, 2.2 с применением вложения (2.17).

Для доказательства вложений типа (2.17) нами будет применяться следующая лемма. Пусть $E_{0}$ и $E_{1}$ - банаховы пространства такие, что $E_{1} \subset E_{0}$. Рассмотрим пространство

$$
\begin{aligned}
& W_{\infty, p_{0}}\left(0, T ; E_{1}, E_{0}\right) \\
& \quad=\left\{\psi(s), s \in[0, T]: \psi(s) \in L_{\infty}\left(0, T ; E_{1}\right), \psi^{\prime}(s) \in L_{p_{0}}\left(0, T ; E_{0}\right)\right\}
\end{aligned}
$$

$\left(p_{0}>1\right)$ с нормой

$$
\|\psi\|_{W_{\infty, p_{0}}}=\operatorname{ess} \sup \left\{\|\psi(s)\|_{E_{1}}: s \in[0, T]\right\}+\left(\int_{0}^{T}\left\|\psi^{\prime}(s)\right\|_{E_{0}}^{p_{0}} d s\right)^{1 / p_{0}}
$$

ЛЕмма 2.1. Предположим, что

$$
E_{1} \Subset E \subset E_{0}
$$

Тогда следующее вложсение компактно:

$$
W_{\infty, p_{0}}\left(0, T ; E_{1}, E_{0}\right) \Subset C([0, T] ; E) .
$$

Эта лемма доказана в приложении.

ЗАмЕчАниЕ 2.2. Предельное поведение решений неавтономных уравнений вида (2.10), для которых задача Коши (2.14) однозначно разрешима, можно описывать с помощью соответствующих глобальных равномерных аттракторов. При этом аналогомполугруппы $\{S(t)\}$ (см. (2.15)) служит понятие процесса $\left\{U_{\sigma}(t, \tau)\right\}$, которьй порождает неавтономное уравнение. Теория процессов и их аттракторов изложена в [10] (см. также [11], [12]). Отметим, что глобальные аттракторы процессов можно строить с помощью равномерных траекторных аттракторов изучаемых неавтономных уравнений, используя теорему 2.3 и очевидное обобшение теоремы 2.4 .

В следующем параграфе будут изучаться эволюционные уравнения и их траекторные и глобальные аттракторы, зависящие от малого параметра $\varepsilon>0$.

ОПРЕДЕЛЕНИЕ 2.4. Будем говорить, что траекторные аттракторы $\mathfrak{A}_{\varepsilon}$ cходяmся к траекторному аттрактору $\overline{\mathfrak{A}}$ при $\varepsilon \rightarrow 0+$ в топологическом пространстве $\Theta_{+}^{\text {loc }}$, если для любой окрестности $\mathscr{O}(\overline{\mathfrak{A}})$ в $\Theta_{+}^{\text {loc }}$ найдется $\varepsilon_{1} \geqslant 0$ такое, что $\mathfrak{A}_{\varepsilon} \subseteq \mathscr{O}(\overline{\mathfrak{A}})$ при любом $\varepsilon<\varepsilon_{1}$. В частности, если $\Theta_{t_{1}, t_{2}}-$ метрические пространства, то

$$
\operatorname{dist}_{\Theta_{t_{1}, t_{2}}}\left(\Pi_{t_{1}, t_{2}} \mathfrak{A}_{\varepsilon}, \Pi_{t_{1}, t_{2}} \overline{\mathfrak{A}}\right) \rightarrow 0 \quad(\varepsilon \rightarrow 0+) .
$$

Аналогично, глобальные аттракторы $\mathscr{A}_{\varepsilon}$ сходятся к глобальному аттрактору $\overline{\mathscr{A}}$ в банаховом пространстве $E$, если

$$
\operatorname{dist}_{E}\left(\mathscr{A}_{\varepsilon}, \overline{\mathscr{A}}\right) \rightarrow 0 \text { при } \varepsilon \rightarrow 0+.
$$




\section{§ 3. Усреднение уравнений и систем}

3.1. Трехмерная система Навье-Стокса. Рассмотрим систему НавьеСтокса в области $\Omega \Subset \mathbb{R}^{3}$ :

$$
\partial_{t} u+\nu L u+B(u)=g\left(x, \frac{x}{\varepsilon}\right), \quad(\nabla, u)=0,\left.u\right|_{\partial \Omega}=0
$$

где $x=\left(x_{1}, x_{2}, x_{3}\right) \in \Omega, u=u(x, t)=\left(u^{1}, u^{2}, u^{3}\right)$ и $g=\left(g^{1}, g^{2}, g^{3}\right)$. Здесь $L$ - оператор Стокса в $\mathbb{R}^{3}: L u=-P \Delta u ; B(u)=B(u, u), B(u, v)=P(u, \nabla) v=$ $P \sum_{i=1}^{3} u_{i} \partial_{x_{i}} v$. Через $H$ и $V$ обозначены замыкания в $\left(L_{2}(\Omega)\right)^{3}$ и $\left(H^{1}(\Omega)\right)^{3}$ множества $\mathscr{V}_{0}=\left\{v: v \in\left(C_{0}^{\infty}(\Omega)\right)^{3},(\nabla, v)=0\right\}$. Символ $P$ обозначает ортогональньй проектор пространства $\left(L_{2}(\Omega)\right)^{3}$ на гильбертово пространство $H$. Для скалярных произведений в $H$ и $V$ используются обозначения: $(u, v)=\int_{\Omega}(u(x), v(x)) d x$ и $((u, v))=\langle L u, v\rangle=\int_{\Omega}(\nabla u(x), \nabla v(x)) d x$, а нормы в этих пространствах обозначаются $|u|=(u, u)^{1 / 2}$ и $\|u\|=\langle L u, u\rangle^{1 / 2}$ соответственно.

Будем предполагать, что $g(x, x / \varepsilon) \in H$ при любом $\varepsilon>0$ и она имеет среднее $\bar{g}(x)$ при $\varepsilon \rightarrow 0+$ в пространстве $H_{w}$, т.е.

$$
\left(g\left(x, \frac{x}{\varepsilon}\right), \varphi(x)\right) \rightarrow(\bar{g}(x), \varphi(x)) \text { при } \varepsilon \rightarrow 0+\forall \varphi \in H .
$$

ЗАМЕчАНИЕ 3.1. Используя $\S 1$, можно построить множество примеров функций $g(x, x / \varepsilon)$, удовлетворяющих (3.2). Например, $g(x, x / \varepsilon)=g_{1}(x) g_{2}(x / \varepsilon)$, где $g_{1} \in H$, а $g_{2}(z)$ - периодическая, квазипериодическая (по $z$ ) функция или просто функция, имеющая среднее $\bar{g}_{2} \in \mathbb{R}$ в $L_{\infty, * w}(\Omega) \cap H$.

Для описания пространства траекторий $\mathscr{K}_{\varepsilon}^{+}$уравнения (3.1) рассмотрим слабые решения этого уравнения в пространстве $L_{2}^{\text {loc }}\left(\mathbb{R}_{+} ; V\right) \cap L_{\infty}^{\text {loc }}\left(\mathbb{R}_{+} ; H\right)$. Если $u(s) \in L_{2}^{\text {loc }}\left(\mathbb{R}_{+} ; V\right) \cap L_{\infty}^{\text {loc }}\left(\mathbb{R}_{+} ; H\right)$, то уравнение $(3.1)$ можно понимать в смысле обобщенных функций пространства $D^{\prime}\left(\mathbb{R}_{+} ; V^{\prime}\right)$, где $V^{\prime}$ - пространство, сопряженное к $V$ (см. [13]).

ОПРЕДЕЛЕНИЕ 3.1. Пространство траекторий $\mathscr{K}_{\varepsilon}^{+}$уравнения (3.1) есть объединение слабых решений $u(s) \in L_{2}^{\text {loc }}\left(\mathbb{R}_{+} ; V\right) \cap L_{\infty}^{\text {loc }}\left(\mathbb{R}_{+} ; H\right)$ этого уравнения, которые удовлетворяют следуюшему неравенству:

$$
\frac{1}{2} \frac{d}{d t}|u(t)|^{2}+\nu\|u(t)\|^{2} \leqslant(g, u(t)), \quad t \in \mathbb{R}_{+} .
$$

Здесь $g=g(x, x / \varepsilon)$. Неравенство (3.3) следует понимать так: для любой функции $\psi(s) \in C_{0}^{\infty}(] 0,+\infty[), \psi \geqslant 0$, справедливо неравенство

$$
-\frac{1}{2} \int_{0}^{+\infty}|u(s)|^{2} \psi^{\prime}(s) d s+\nu \int_{0}^{+\infty}\|u(s)\|^{2} \psi(s) d s \leqslant \int_{0}^{+\infty}(g, u(s)) \psi(s) d s
$$

Если $u_{0} \in H$, то сушествует слабое решение $u(s)$ уравнения $(3.1)$, принадлежащее пространству $L_{2}^{\text {loc }}\left(\mathbb{R}_{+} ; V\right) \cap L_{\infty}^{\text {loc }}\left(\mathbb{R}_{+} ; H\right)$, такое, что $u(0)=u_{0}$ и $u(s)$ удовлетворяет неравенству (3.4). Для доказательства см. [13], [6]. 
ЗАмечАниЕ 3.2. Для трехмерной системы Навье-Стокса проблема единственности слабого решения остается открытой. Неизвестно также, будет ли любое слабое решение (3.1) удовлетворять неравенству (3.3). Однако любые слабые решения $u(t), t \geqslant 0$, которые получены методом галёркинских приближений, удовлетворяют (3.3).

Известно, что для любого слабого решения $u(s) \in L_{2}^{\text {loc }}\left(\mathbb{R}_{+} ; V\right) \cap L_{\infty}^{\text {loc }}\left(\mathbb{R}_{+} ; H\right)$ уравнения (3.1) производная $\partial_{t} u \in L_{4 / 3}^{\text {loc }}\left(\mathbb{R}_{+} ; V^{\prime}\right)$. Рассмотрим пространство

$$
\mathscr{F}_{+}^{\text {loc }}=L_{2}^{\text {loc }}\left(\mathbb{R}_{+} ; V\right) \cap L_{\infty}^{\text {loc }}\left(\mathbb{R}_{+} ; H\right) \cap\left\{v: \partial_{t} v \in L_{4 / 3}^{\text {loc }}\left(\mathbb{R}_{+} ; V^{\prime}\right)\right\}
$$

снабженное следуюшей топологией сходимости. Последовательность $\left\{v_{n}\right\} \subset \mathscr{F}_{+}^{\text {loc }}$

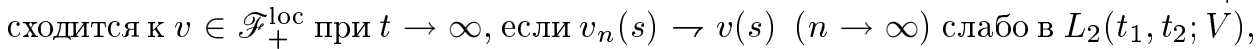
*-слабо в $L_{\infty}\left(t_{1}, t_{2} ; H\right)$ и $\partial_{t} v_{n}(s) \rightarrow \partial_{t} v(s)(n \rightarrow \infty)$ слабо в $L_{4 / 3}\left(t_{1}, t_{2} ; V^{\prime}\right)$ для каждого интервала $\left[t_{1}, t_{2}\right] \subset \mathbb{R}_{+}$. Пространство $\mathscr{F}_{+}^{\text {loc }}$, снабженное такой слабой топологией, обозначается через $\Theta_{+}^{\text {loc }}$. Будем также использовать пространство

$$
\mathscr{F}_{+}^{b}=L_{2}^{b}\left(\mathbb{R}_{+} ; V\right) \cap L_{\infty}^{b}\left(\mathbb{R}_{+} ; H\right) \cap\left\{v: \partial_{t} v \in L_{4 / 3}^{b}\left(\mathbb{R}_{+} ; V^{\prime}\right)\right\}
$$

которое является подпространством пространства $\mathscr{F}_{+}^{\text {loc }}$. Напомним, что

$$
\|v\|_{L_{p}^{b}\left(\mathbb{R}_{+} ; E\right)}=\sup _{h \in \mathbb{R}_{+}}\|v\|_{L_{p}(h, h+1 ; E)}
$$

УТВЕРЖДЕНИЕ 3.1. Для любого $u(s) \in \mathscr{K}_{\varepsilon}^{+}$справедливо неравенство:

$$
\|T(t) u(\cdot)\|_{\mathscr{F}_{+}^{b}} \leqslant C\|u(0)\|_{H}^{2} \exp (-\lambda t)+R_{0} \quad \forall t \geqslant 0
$$

әде $\lambda$ - первое собственное значение оператора $\nu L ; C$ зависит от $\lambda$, a $R_{0}$ зависит от $\lambda u\|g\|_{H}^{2}$ (см. [6]).

На пространстве траекторий $\mathscr{K}_{\varepsilon}^{+}$действует полугруппа сдвигов $\{T(t), t \geqslant 0\}$ :

$$
T(t) u(s)=u(t+s), \quad s \geqslant 0
$$

Очевидно,

$$
T(t) u(s) \in \mathscr{K}_{\varepsilon}^{+} \quad \forall u \in \mathscr{K}_{\varepsilon}^{+}, \quad t \geqslant 0 .
$$

Следовательно,

$$
T(t) \mathscr{K}_{\varepsilon}^{+} \subseteq \mathscr{K}_{\varepsilon}^{+} \quad \forall t \geqslant 0
$$

Из неравенства (3.5) следует, что шар $B_{0}=\|v\|_{\mathscr{F}_{+}^{b}} \leqslant 2 R_{0}$ является поглощающим множеством полугруппы $\{T(t)\}$ на $\mathscr{K}_{\varepsilon}^{+}$. Множество $B_{0}$ ограничено в $\mathscr{F}_{+}^{b}$ и компактно в $\Theta_{+}^{\text {loc }}$. 
УТВЕРЖДЕНИЕ 3.2. Пространство траекторий $\mathscr{K}_{\varepsilon}^{+}$замкнуто в $\Theta_{+}^{\text {loc }}$ $(c \mathcal{M} .[6])$.

Ядро $\mathscr{K}_{\varepsilon}$ уравнения (3.1) состоит из всех его слабых решений $u(s), s \in \mathbb{R}$, удовлетворяющих неравенству (3.4) при любой $\psi(s) \in C_{0}^{\infty}(\mathbb{R}), \psi \geqslant 0$, и ограниченных в пространстве

$$
\mathscr{F}^{b}=L_{2}^{b}(\mathbb{R} ; V) \cap L_{\infty}(\mathbb{R} ; H) \cap\left\{v: \partial_{t} v \in L_{4 / 3}^{b}\left(\mathbb{R} ; V^{\prime}\right)\right\} .
$$

В силу утверждений 3.1 и 3.2 применимы теоремы 2.1 и 2.2. Уравнение (3.1) имеет траекторный аттрактор $\mathfrak{A}_{\varepsilon}$. Множество $\mathfrak{A}_{\varepsilon}$ ограничено в $\mathscr{F}_{+}^{b}$ и компактно в $\Theta_{+}^{\text {loc }}$. Кроме того,

$$
\mathscr{A}_{\varepsilon}=\Pi_{+} \mathscr{K}_{\varepsilon}
$$

ядро $\mathscr{K}_{\varepsilon}$ не пусто, ограничено в $\mathscr{F}^{b}$ и компактно в $\Theta^{\text {loc }}$.

Заметим, что имеют место следующие (непрерывные) вложения:

$$
\begin{aligned}
& \Theta_{+}^{\text {loc }} \subset L_{2}^{\text {loc }}\left(\mathbb{R}_{+} ; H^{1-\delta}\right), \\
& \Theta_{+}^{\text {loc }} \subset C\left(\mathbb{R}_{+} ; H^{-\delta}\right), \quad 0<\delta \leqslant 1 .
\end{aligned}
$$

Вложение (3.8) получается из леммы 2.1 и вложений $H \Subset H^{-\delta} \subset V^{\prime}$, если положить: $E_{0}=V^{\prime}, E=H^{-\delta}, E_{1}=H$ и $p_{0}=4 / 3$. Вложение (3.7) вытекает из аналогичной леммы о компактном вложении в $L_{p}(0, M ; E)$ (см. $[13 ;$ гл. 1 , теорема 5.1]).

Следовательно, для любого ограниченного (в $\left.\mathscr{F}_{+}^{b}\right)$ множества $B \subset \mathscr{K}_{\varepsilon}^{+}$

$$
\begin{aligned}
\operatorname{dist}_{L_{2}\left(0, M ; H^{1-\delta}\right)}\left(\Pi_{0, M} T(t) B, \Pi_{0, M} \mathscr{K}_{\varepsilon}\right) \rightarrow 0 & (t \rightarrow \infty), \\
\operatorname{dist}_{C\left([0, M] ; H^{-\delta}\right)}\left(\Pi_{0, M} T(t) B, \Pi_{0, M} \mathscr{K}_{\varepsilon}\right) \rightarrow 0 & (t \rightarrow \infty),
\end{aligned}
$$

где $M$ - произвольное положительное число.

Одновременно с уравнением (3.1) рассмотрим усредненное уравнение

$$
\partial_{t} \bar{u}+\nu L \bar{u}+B(\bar{u})=\bar{g}(x), \quad(\nabla, \bar{u})=0,\left.\quad \bar{u}\right|_{\partial \Omega}=0 .
$$

Ясно, что уравнение (3.9) также имеет траекторный аттрактор $\overline{\mathfrak{A}}$ в соответствующем пространстве траекторий $\overline{\mathscr{K}}^{+}$, отвечающем уравнению (3.9) (см. определение 3.1$)$, и

$$
\overline{\mathfrak{A}}=\Pi_{+} \overline{\mathscr{K}}
$$

где $\overline{\mathscr{K}}$ - ядро уравнения (3.9) в $\mathscr{F} b$. Сформулируем основную теорему.

Теорема 3.1. Имеет место следующее предельное соотношение в пространстве $\Theta_{+}^{\text {loc }}$ :

$$
\mathfrak{A}_{\varepsilon} \rightarrow \overline{\mathfrak{A}} \quad \text { npu } \quad \varepsilon \rightarrow 0+.
$$

Кроме того,

$$
\mathscr{K}_{\varepsilon} \rightarrow \overline{\mathscr{K}} \quad \text { npu } \quad \varepsilon \rightarrow 0+\quad \text { в } \quad \Theta^{\mathrm{loc}} .
$$


ДокаЗАТЕЛьство. Заметим сразу, что из (3.12) следует (3.11). Поэтому достаточно доказать $(3.12)$, т.е. для любой окрестности $\mathscr{O}(\overline{\mathscr{K}})$ в $\Theta^{\text {loc }}$ найдется $\varepsilon_{1}=$ $\varepsilon_{1}(\mathscr{O})>0$ такое, что

$$
\mathscr{K}_{\varepsilon} \subset \mathscr{O}(\overline{\mathscr{K}}) \text { для } \varepsilon<\varepsilon_{1} .
$$

Предположим, что (3.13) не верно. Тогда существуют окрестность $\mathscr{O}^{\prime}(\overline{\mathscr{K}})$ в $\Theta^{\text {lос }}$, последовательность $\varepsilon_{n} \rightarrow 0+(n \rightarrow \infty)$ и последовательность $u_{\varepsilon_{n}}(s) \in \mathscr{K}_{\varepsilon_{n}}^{+}$такие, чTо

$$
u_{\varepsilon_{n}} \notin \mathscr{O}^{\prime}(\overline{\mathscr{K}}) \quad \forall n \in \mathbb{N} .
$$

Из условия (3.2) вытекает, что последовательность $\left\{g\left(x, x / \varepsilon_{n}\right)\right\}$ ограничена в $H$. Следовательно, из предложения 3.1 заключаем, что последовательность $\left\{u_{\varepsilon_{n}}\right\}$ ограничена в $\mathscr{F}^{b}$. Переходя к подпоследовательности, мы можем предположить, что

$$
u_{\varepsilon_{n}} \rightarrow \bar{u}(n \rightarrow \infty) \quad \text { в } \quad \Theta^{1 \mathrm{loc}}
$$

У тверждается, что $\bar{u} \in \overline{\mathscr{K}}$. Функции $u_{\varepsilon_{n}}(x, s)$ удовлетворяют уравнению

$$
\partial_{t} u_{\varepsilon_{n}}+\nu L u_{\varepsilon_{n}}+B\left(u_{\varepsilon_{n}}\right)=g\left(x, \frac{x}{\varepsilon_{n}}\right), \quad t \in \mathbb{R}
$$

и неравенству

$$
\begin{aligned}
& -\frac{1}{2} \int_{-M}^{M}\left|u_{\varepsilon_{n}}(s)\right|^{2} \psi^{\prime}(s) d s+\nu \int_{-M}^{M}\left\|u_{\varepsilon_{n}}(s)\right\|^{2} \psi(s) d s \\
& \quad \leqslant \int_{-M}^{M}\left(g\left(x, \frac{x}{\varepsilon_{n}}\right), u_{\varepsilon_{n}}(s)\right) \psi(s) d s
\end{aligned}
$$

при любом $M>0$ и для любой функции $\psi \in C_{0}^{\infty}(]-M, M[), \psi \geqslant 0$. Кроме того, $u_{\varepsilon_{n}}(s) \rightarrow \bar{u}(s)(n \rightarrow \infty)$ слабо в $L_{2}(-M, M ; V), *$-слабо в $L_{\infty}(-M, M ; H)$ и $\partial_{t} u_{\varepsilon_{n}}(s) \rightarrow \partial_{t} \bar{u}(s)(n \rightarrow \infty)$ слабо в $L_{4 / 3}\left(-M, M ; V^{\prime}\right)$. В силу известной теоремы о компактности из [13] мы можем также считать, что $u_{\varepsilon_{n}}(s) \rightarrow \bar{u}(s)$ $(n \rightarrow \infty)$ сильно в $L_{2}(-M, M ; H)$ и $u_{\varepsilon_{n}}(x, s) \rightarrow \bar{u}(x, s)(n \rightarrow \infty)$ при почти всех $(x, s) \in \Omega \times]-M, M\left[\right.$. В силу (3.2) имеем, что $g\left(x, x / \varepsilon_{n}\right) \rightarrow \bar{g}(x)(n \rightarrow \infty)$ слабо в $H_{w}$ и, следовательно, слабо в $L_{2}(-M, M ; H)$. Теперь мы можем совершить предельный переход в (3.15) и (3.16), используя стандартное рассуждение из [13] (см. подробное доказательство в [6]). Следовательно, $\bar{u} \in \mathscr{K}_{\bar{g}} \equiv \overline{\mathscr{K}}$, т.е. $\bar{u}-$ решение (3.9), удовлетворяюшее соответствуюшему неравенству (3.16) с внешней силой $\bar{g}(x)$. В то же время мы установили, что $u_{\varepsilon_{n}}(s) \rightarrow \bar{u}(s)(n \rightarrow \infty)$ в $\Theta_{+}^{\text {loc }}$ и, следовательно, $u_{\varepsilon_{n}}(s) \in \mathscr{O}^{\prime}(\bar{u}(s)) \subset \mathscr{O}^{\prime}(\overline{\mathscr{K}})$ при $\varepsilon_{n} \ll 1$. Это противоречит (3.14). Теорема доказана.

Применяя вложения (3.7) и (3.8), получаем

СлЕДСТВИЕ 3.1. Для любого $0<\delta \leqslant 1$ и ююбого $M>0$

$$
\begin{aligned}
\operatorname{dist}_{L_{2}\left(0, M ; H^{1-\delta}\right)}\left(\Pi_{0, M} \mathfrak{A}_{\varepsilon}, \Pi_{0, M} \overline{\mathfrak{A}}\right) \rightarrow 0 & (\varepsilon \rightarrow 0+), \\
\operatorname{dist}_{C\left([0, M] ; H^{-\delta}\right)}\left(\Pi_{0, M} \mathfrak{A}_{\varepsilon}, \Pi_{0, M} \overline{\mathfrak{A}}\right) \rightarrow 0 & (\varepsilon \rightarrow 0+) .
\end{aligned}
$$


Теперь мы кратко рассмотрим трехмерную неавтономную систему Навье-Стокса, содержашую внешнюю силу, которая быстро осциллирует по времени. Система имеет вид

$$
\partial_{t} u+\nu L u+B(u)=g\left(x, t, \frac{t}{\varepsilon}\right), \quad(\nabla, u)=0,\left.u\right|_{\partial \Omega}=0
$$

Для простоты предполагается, что $g(x, t, t / \varepsilon)=P g_{1}(x, t / \varepsilon) g_{2}(x, t)$, где $g_{1}(x, s)-$ тр.-к. функция в $L_{2}^{\text {loc }}\left(\mathbb{R} ; L_{2}(\Omega)^{3}\right)$, а $g_{2}(x, s)$ тр.-к. в $C_{b}\left(\mathbb{R} ; C(\bar{\Omega})^{3}\right)$. Легко доказывается, что функция $P g_{1}(x, t / \varepsilon) g_{2}(x, t)$ трансляционно-компактна в $L_{2}^{\text {loc }}(\mathbb{R} ; H)$ при любом фиксированном $\varepsilon>0$. Пусть также функция $g_{1}(x, t / \varepsilon)$ имеет равномерное среднее по времени в $L_{2, w}^{\text {loc }}(\mathbb{R} ; H)$. Например, в силу теоремы 1.5 достаточно предположить, что

$$
\frac{1}{\mu} \int_{h}^{h+\mu} g_{1}(x, s) d s \rightarrow \bar{g}_{1}(x) \quad(\mu \rightarrow \infty) \text { сильно в } H
$$

равномерно по $h \in \mathbb{R}$. Аналогично примеру 1.6 легко доказывается, что функция $g_{\varepsilon}(x, t) \equiv g(x, t, t / \varepsilon)$ имеет равномерное среднее $\bar{g}(x, t) \equiv P \bar{g}_{1}(x) g_{2}(x, t)$ в $L_{2, w}^{\mathrm{loc}}(\mathbb{R} ; H)$ при $\varepsilon \rightarrow 0+$. Пусть $\mathscr{H}_{+}\left(g_{\varepsilon}(x, t)\right)$ и $\mathscr{H}_{+}(\bar{g}(x, t))$ - оболочки функций $g(x, t, t / \varepsilon)$ и $\bar{g}(x, t)$ в $L_{2}^{\text {loc }}(\mathbb{R} ; H)$. Подобно примеру 1.6 доказывается, что в $L_{2, w}^{\operatorname{loc}}(\mathbb{R} ; H)$

$$
\mathscr{H}\left(g_{\varepsilon}(x, t)\right) \rightarrow \mathscr{H}(\bar{g}(x, t)) \quad(\varepsilon \rightarrow 0+) .
$$

Усредненной внешней силе соответствует усредненное уравнение

$$
\partial_{t} \bar{u}+\nu L \bar{u}+B(\bar{u})=\bar{g}(x, t), \quad(\nabla, \bar{u})=0,\left.\quad \bar{u}\right|_{\partial \Omega}=0 .
$$

Следуя схеме, изложенной в $\S 2$ применительно к абстрактному неавтономному уравнению $(2.10)$, определяются пространства $\mathscr{K}_{\mathscr{H}\left(g_{\varepsilon}\right)}^{+}$и $\mathscr{K}_{\mathscr{H}(\bar{g})}^{+}$для уравнений (3.18) и (3.21) соответственно. Эти уравнения по теореме 2.3 имеют равномерные траекторные аттракторы $\mathfrak{A}_{\mathscr{H}\left(g_{\varepsilon}\right)}$ и $\mathfrak{A}_{\mathscr{H}(\bar{g})}$. Имеет место следующий результат.

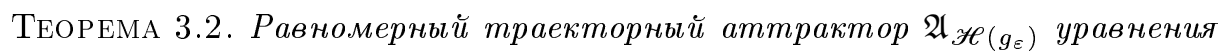
(3.18) стремится к равномерному траекторному аттрактору $\mathfrak{A}_{\mathscr{H}(\bar{g})}$ усредненного уравнения (3.21) при $\varepsilon \rightarrow 0+$ в пространстве $\Theta_{+}^{\text {loc }}$.

Доказательство этой теоремы аналогично доказательству теоремы 3.1. Используется обобщение утверждения 3.1 и применяется предел (3.20).

СлЕДСТВИЕ 3.2. В силу вложсний:

$$
\left.\left.\Theta_{+}^{\text {loc }} \subset L_{2}\left(0, M ; H^{1-\delta}\right), \quad \Theta_{+}^{\text {loc }} \subset C\left([0, M] ; H^{-\delta}\right) \quad(\delta \in] 0,1\right]\right)
$$

мьц заключаем, что при любом $M>0$

$$
\begin{aligned}
\operatorname{dist}_{L_{2}\left(0, M ; H^{1-\delta}\right)}\left(\Pi_{0, M} \mathfrak{A}_{\mathscr{H}\left(g_{\varepsilon}\right)}, \Pi_{0, M} \mathfrak{A}_{\mathscr{H}(\bar{g})}\right) \rightarrow 0 & (\varepsilon \rightarrow 0+), \\
\operatorname{dist}_{C\left([0, M] ; H^{-\delta}\right)}\left(\Pi_{0, M} \mathfrak{A}_{\mathscr{H}\left(g_{\varepsilon}\right)}, \Pi_{0, M} \mathfrak{A}_{\mathscr{H}(\bar{g})}\right) \rightarrow 0 & (\varepsilon \rightarrow 0+) .
\end{aligned}
$$


3.2. Двумерная система Навье-Стокса. Все изложенные выше результаты применимы также к двумерной системе Навье-Стокса. В этом пункте доказывается теорема об усреднении двумерной системы для случая, когда внешняя сила осциллирует более сингулярно и имеет среднее только в пространстве $V^{\prime}$ (а не в $H$, как это было в предыдущем пункте).

Аналогично трехмерному случаю доказывается существование траекторного аттрактора $\mathfrak{A}_{\varepsilon}$ для следуюшей системы Навье-Стокса в $\mathbb{R}^{2}$ :

$$
\partial_{t} u+\nu L u+B(u)=g_{0}\left(x, \frac{x}{\varepsilon}\right)+P \partial_{x_{1}} g_{1}\left(x, \frac{x}{\varepsilon}\right)+P \partial_{x_{2}} g_{2}\left(x, \frac{x}{\varepsilon}\right),\left.u\right|_{\partial \Omega}=0,
$$

где $x \in \Omega \Subset \mathbb{R}^{2}$ и $g_{i}(x, x / \varepsilon) \in H$ при $i=0,1,2$. Ясно, что правая часть (3.22) принадлежит $V^{\prime}$. Напомним, что траекторньй аттрактор $\mathfrak{A}_{\varepsilon}$ притягивает ограниченные множества из $\mathscr{K}_{\varepsilon}^{+}$в топологии

$$
\Theta_{+}^{\text {loc }}=L_{2, w}^{\text {loc }}\left(\mathbb{R}_{+} ; V\right) \cap L_{\infty, * w}^{\text {loc }}\left(\mathbb{R}_{+} ; H\right) \cap\left\{v: \partial_{t} v \in L_{2, w}^{\text {loc }}\left(\mathbb{R}_{+} ; V^{\prime}\right)\right\},
$$

причем ограниченные множества из $\mathscr{K}_{\varepsilon}^{+}$берутся по норме пространства

$$
\mathscr{F}_{+}^{b}=L_{2}^{b}\left(\mathbb{R}_{+} ; V\right) \cap L_{\infty}^{b}\left(\mathbb{R}_{+} ; H\right) \cap\left\{v: \partial_{t} v \in L_{2}^{b}\left(\mathbb{R}_{+} ; V^{\prime}\right)\right\} .
$$

Предполагается, что функции $g_{i}(x, x / \varepsilon)$ имеют средние $\bar{g}_{i}(x), i=0,1,2$, в $H_{w}$ при $\varepsilon \rightarrow 0+$. Следовательно, функция $g(x, x / \varepsilon)=g_{0}(x, x / \varepsilon)+P \partial_{x_{1}} g_{1}(x, x / \varepsilon)+$ $P \partial_{x_{2}} g_{2}(x, x / \varepsilon)$ имеет среднее $\bar{g}(x)=\bar{g}_{0}(x)+P \partial_{x_{1}} \bar{g}_{1}(x)+P \partial_{x_{2}} \bar{g}_{2}(x)$ в $V_{w}$, т.е.

$$
\left\langle g\left(x, \frac{x}{\varepsilon}\right), \varphi(x)\right\rangle \rightarrow\langle\bar{g}(x), \varphi(x)\rangle \text { при } \varepsilon \rightarrow 0+\forall \varphi \in V .
$$

Заметим, что функции $\partial_{x_{i}} g_{i}(x, x / \varepsilon)=g_{i x_{i}}(x, x / \varepsilon)+(1 / \varepsilon) g_{i z_{i}}(x, x / \varepsilon)$ могут иметь неограниченно растущие нормы в $H$ при $\varepsilon \rightarrow 0+$.

Усредненное уравнение

$$
\partial_{t} \bar{u}+\nu L \bar{u}+B(\bar{u})=\bar{g}_{0}(x)+P \partial_{x_{1}} \bar{g}_{1}(x)+P \partial_{x_{2}} \bar{g}_{2}(x),\left.\quad \bar{u}\right|_{\partial \Omega}=0,
$$

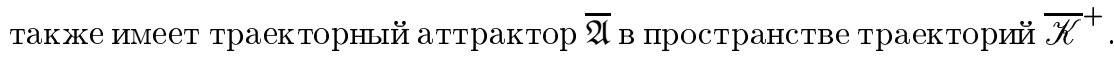

Аналогично теореме 3.1 доказывается

Tеорема 3.3. B топологии $\Theta_{+}^{\mathrm{loc}}$ имеем:

$$
\mathfrak{A}_{\varepsilon} \rightarrow \overline{\mathfrak{A}} \quad \text { npu } \quad \varepsilon \rightarrow 0+.
$$

Мы не приводим здесь этого доказательства. Отметим лишш, что в уравнении (3.22) можно сделать предельньй переход, если известно, что

$$
g\left(x, \frac{x}{\varepsilon}\right) \rightarrow \bar{g}(x) \text { при } \varepsilon \rightarrow 0+\text { в } V^{\prime} .
$$

Рассмотрим также случай, когда в уравнениях (3.22) и $(3.23) g_{1} \equiv g_{2} \equiv 0$, т.е. функция $g(x, x / \varepsilon)=g_{0}(x, x / \varepsilon) \in H$ имеет в $H_{w}$ среднее $\bar{g}(x)=\bar{g}_{0}(x) \in$ $H$. В работе [14] доказано, что траекторные аттракторы $\mathfrak{A}_{\varepsilon}$ и $\overline{\mathfrak{A}}$ этих уравнений существуют в более сильном топологическом пространстве $\Theta_{+}^{\text {loc }}=H_{w}^{\mathbf{r}, \text { loc }}, \mathbf{r}=$ $(2,2,1)$, где

$$
H_{w}^{\mathbf{r}, \mathrm{loc}}=L_{2, w}^{\mathrm{loc}}\left(\mathbb{R}_{+} ; H^{2}\right) \cap\left\{v: \partial_{t} v \in L_{2, w}^{\mathrm{loc}}\left(\mathbb{R}_{+} ; H\right)\right\} .
$$

С помощью более сильных априорных оценок для уравнения (3.22) при $g_{1} \equiv g_{2} \equiv 0$ установлен следующий результат. 
ТЕОРема 3.4. Предел (3.24) имеет место в топологии $\Theta_{+}^{\text {loc }}=H_{w}^{\mathbf{r}, \text { loc }}$.

Заметим, что топология пространства $H_{w}^{\mathbf{r}, \text { loc }}$ сильнее топологии локальной равномерной сходимости пространства $C^{\mathrm{loc}}\left(\mathbb{R}_{+} ; H^{1-\delta}\right): H_{w}^{\mathbf{r}}\left(Q_{M}\right) \subset C\left([0, M] ; H^{1-\delta}\right)$ при $\delta \in] 0,1]$. Отсюда вытекает

СЛЕДСТВИЕ 3.3. Выполнено предельное соотношение:

$$
\operatorname{dist}_{C\left([0, M] ; H^{1-\delta}\right)}\left(\Pi_{M} \mathfrak{A}_{\varepsilon}, \Pi_{M} \overline{\mathfrak{A}}\right) \rightarrow 0 \quad(\varepsilon \rightarrow 0+) \quad \forall M \geqslant 0
$$

В заключение докажем один результат об усреднении глобального аттрактора двумерной системы Навье-Стокса. Хорошо известно, что система $(3.22)$ с внешней силой $g(x, x / \varepsilon)=g_{0}(x, x / \varepsilon) \in H$ при фиксированном $\varepsilon>0$ порождает полугруппу $\left\{S_{\varepsilon}(t)\right\}$, действующую в фазовом пространстве $H$, которая имеет глобальный аттрактор $\mathscr{A}_{\varepsilon} \Subset V, S(t) \mathscr{A}_{\varepsilon}=\mathscr{A}_{\varepsilon}$ при $t \geqslant 0$ (см., например, [7], [8]). Усредненное уравнение с усредненной внешней силой $\bar{g}(x)=\bar{g}_{0}(x) \in H$ также имеет глобальньй аттрактор $\overline{\mathscr{A}} \Subset V$. Применима теорема 2.4. Имеем:

$$
\mathscr{A}_{\varepsilon}=\left\{u(0): u \in \mathfrak{A}_{\varepsilon}\right\} \quad \text { и } \overline{\mathscr{A}}=\{u(0): u \in \overline{\mathfrak{A}}\} .
$$

Из (3.25) вытекает

СлЕДСТВИЕ 3.4. Если функиия $g(x, x / \varepsilon)$ имеет среднее $\bar{g}(x)$ в $H_{w}$ nри $\varepsilon \rightarrow 0+, m o$

$$
\left.\left.\operatorname{dist}_{H^{1-\delta}}\left(\mathscr{A}_{\varepsilon}, \overline{\mathscr{A}}\right) \rightarrow 0 \quad(\varepsilon \rightarrow 0+), \quad \delta \in\right] 0,1\right]
$$

Аналогичные результаты справедливы для неавтономных двумерных систем Навье-Стокса с внешними силами, быстро осциллирующими по времени.

3.3. Системы уравнений реакции-диффузии. Рассматривается система реакции-диффузии с быстро осциллирующими членами вида

$$
\partial_{t} u=a \Delta u-b\left(x, \frac{x}{\varepsilon}\right) f(u)+g\left(x, \frac{x}{\varepsilon}\right),\left.\quad u\right|_{\partial \Omega}=0
$$

где $x \in \Omega \Subset \mathbb{R}^{n}, u=\left(u^{1}, \ldots, u^{N}\right), f=\left(f^{1}, \ldots, f^{N}\right)$ и $g=\left(g^{1}, \ldots, g^{N}\right)$. Здесь $a$ обозначает $(N \times N)$-матрицу с положительной симметричной частью и $b(x, z) \in$ $C\left(\Omega \times \mathbb{R}^{N}\right)$ - вещественная положительная функция.

Сразу отметим, что предлагаемыми методами можно исследовать системы, у которых нелинейные члены имеют вид $\sum_{j=1}^{m} b_{j}(x, x / \varepsilon) f_{j}(u)$, где $b_{j}$ - матрицы, a $f_{j}(u)$ - векторы-многочлены по $u$. Для краткости мы рассмотрим случай $m=1$ и $b_{1}(x, x / \varepsilon)=b(x, x / \varepsilon) I$, где $I-$ единичная матрица.

Для простоты предположим, что вектор-функция $f(v) \in C\left(\mathbb{R}^{N} ; \mathbb{R}^{N}\right)$ удовлетворяет следующим неравенствам:

$$
f(v) \cdot v \geqslant \gamma|v|^{p}-C, \quad|f(v)| \leqslant C_{1}\left(|v|^{p-1}+1\right), \quad p \geqslant 2
$$


Допускаются также другие условия, например неравенства с различными степенями $\mathbf{p}=\left(p_{1}, \ldots, p_{2}\right)$ вида

$$
\begin{gathered}
f(v) \cdot v \geqslant \gamma \sum_{i=1}^{N}\left|v^{i}\right|^{p_{i}}-C \\
\sum_{i=1}^{N}\left|f^{i}(v)\right|^{p_{i} /\left(p_{i}-1\right)} \leqslant C_{1}\left(\sum_{i=1}^{N}\left|v^{i}\right|^{p_{i}}+1\right), \quad p_{k} \geqslant 2, \quad \forall v \in \mathbb{R}^{N}
\end{gathered}
$$

Заметим, что выполнение условия Липшица для функции $f(v)$ относительно $v$ не предполагается.

Мы будем также считать, что $b(x, z) \in C_{b}(\bar{\Omega} \times \mathbb{R})$ и

$$
\beta_{1} \geqslant b(x, z) \geqslant \beta_{0}>0 \quad \forall x \in \Omega, \quad z \in \mathbb{R}^{n},
$$

а функция $b(x, x / \varepsilon)$ имеет среднее $\bar{b}(x)$ при $\varepsilon \rightarrow 0+$ в $L_{\infty, * w}(\Omega)$, т.е.

$$
\int_{\Omega} b\left(x, \frac{x}{\varepsilon}\right) \varphi(x) d x \rightarrow \int_{\Omega} \bar{b}(x) \varphi(x) d x \quad(\varepsilon \rightarrow 0+)
$$

для любой функции $\varphi \in L_{1}(\Omega)$. Относительно вектор-функции $g(x, x / \varepsilon)$ предполагается, что она имеет среднее $\bar{g}(x)$ в пространстве $V^{\prime}=\left(H^{-1}(\Omega)\right)^{N}$ :

$$
g\left(x, \frac{x}{\varepsilon}\right) \rightarrow \bar{g}(x) \quad(\varepsilon \rightarrow 0+) \text { слабо в } V^{\prime},
$$

т.e.

$$
\left\langle g\left(x, \frac{x}{\varepsilon}\right), \varphi(x)\right\rangle \rightarrow\langle\bar{g}(x), \varphi(x)\rangle \quad(\varepsilon \rightarrow 0+)
$$

для любой $\varphi \in V=\left(H_{0}^{1}(\Omega)\right)^{N}$. В частности, допускаются следующие функции:

$$
g\left(x, \frac{x}{\varepsilon}\right)=g_{0}\left(x, \frac{x}{\varepsilon}\right)+\sum_{i=1}^{n} \partial_{x_{i}} g_{i}\left(x, \frac{x}{\varepsilon}\right)
$$

где функции $g_{i}(x, x / \varepsilon)$ имеют средние $\bar{g}_{i}(x) \in\left(L_{2}(\Omega)\right)^{N}$ в $H=\left(L_{2}(\Omega)\right)^{N}$ :

$$
\left\langle g_{i}\left(x, \frac{x}{\varepsilon}\right), \varphi(x)\right\rangle \rightarrow\left\langle\bar{g}_{i}(x), \varphi(x)\right\rangle \quad(\varepsilon \rightarrow 0+) \quad \forall \varphi \in H, \quad i=1, \ldots, n .
$$

Таким образом, возможен неограниченный рост $H$-норм функций $\partial_{x_{i}} g_{i}(x, x / \varepsilon)=$ $g_{i x_{i}}(x, x / \varepsilon)+(1 / \varepsilon) g_{i z_{i}}(x, x / \varepsilon)$ при $\varepsilon \rightarrow 0+$. Эти функции ограничены лишш в пространстве $V^{\prime}$.

Как и в работе [15], будем исследовать слабые решения системы (3.26), т.е. функции $u(x, s) \in \mathscr{F}_{+}^{\text {loc }}=L_{\infty}^{\text {loc }}\left(\mathbb{R}_{+} ; H\right) \cap L_{2}^{\text {loc }}\left(\mathbb{R}_{+} ; V\right) \cap L_{p}^{\text {loc }}\left(\mathbb{R}_{+} ;\left(L_{p}(\Omega)\right)^{N}\right)$, которые удовлетворяют системе (3.26) в смысле обобшенных функций пространства $D^{\prime}\left(\mathbb{R}_{+} ;\left(H^{-r}(\Omega)\right)^{N}\right)$, где $r=\max \{1 ; n(1 / 2-1 / p)\}$. Для любой $u_{0} \in H$ существует по крайней мере одно слабое решение $u(x, s)$ системы $(3.26)$ такое, что $u(0)=u_{0}$ (см. [7], [15]). Это решение может быть не единственным, поскольку не 
предполагается выполнение условия Липшица для функции $f(v)$ относительно $v$. Обозначим через $\mathscr{K}_{\varepsilon}^{+}$множество всех слабых решений системы (3.26).

Изучается траекторный аттрактор $\mathfrak{A}_{\varepsilon}$ системы (3.26), который по определению совпадает с глобальным $\left(\mathscr{F}_{+}^{b}, \Theta_{+}^{\text {loc }}\right)$-аттрактором полугруппы сдвигов $\{T(t)\}$, действующей на $\mathscr{K}_{\varepsilon}^{+}$. Здесь

$$
\begin{gathered}
\Theta_{+}^{\text {loc }}=L_{\infty, * w}^{\text {loc }}\left(\mathbb{R}_{+} ; H\right) \cap L_{2, w}^{\text {loc }}\left(\mathbb{R}_{+} ; V\right) \cap L_{p, w}^{\text {loc }}\left(\mathbb{R}_{+} ;\left(L_{p}(\Omega)\right)^{N}\right) \\
\cap\left\{v: \partial_{t} v \in L_{q, w}^{\text {loc }}\left(\mathbb{R}_{+} ;\left(H^{-r}(\Omega)\right)^{N}\right)\right\}, \\
\mathscr{F}_{+}^{b}=L_{\infty}^{b}\left(\mathbb{R}_{+} ; H\right) \cap L_{2}^{b}\left(\mathbb{R}_{+} ; V\right) \cap L_{p}^{b}\left(\mathbb{R}_{+} ;\left(L_{p}(\Omega)\right)^{N}\right) \\
\cap\left\{v: \partial_{t} v \in L_{q}^{b}\left(\mathbb{R}_{+} ;\left(H^{-r}(\Omega)\right)^{N}\right)\right\} .
\end{gathered}
$$

Вместе с уравнением (3.26) рассматривается усредненное уравнение

$$
\partial_{t} \bar{u}=a \Delta \bar{u}-\bar{b}(x) f(\bar{u})+\bar{g}(x),\left.\quad \bar{u}\right|_{\partial \Omega}=0,
$$

для которого траекторньй аттрактор $\overline{\mathfrak{A}}$ строится в соответствующем пространстве траекторий $\overline{\mathscr{K}}+$.

УТВЕРЖДЕНИЕ 3.3. При выполнении условий (3.27), (3.29) и (3.30) уравнения (3.26) и (3.31) имеют траекторные аттракторы $\mathfrak{A}_{\varepsilon}$ и $\overline{\mathfrak{A}}$ соответственно. Кроме того,

$$
\mathfrak{A}_{\varepsilon}=\Pi_{+} \mathscr{K}_{\varepsilon} \quad u \quad \overline{\mathfrak{A}}=\Pi_{+} \overline{\mathscr{K}},
$$

әде $\mathscr{K}_{\varepsilon}$ и $\overline{\mathscr{K}}$ обозначают ядра әтих уравнений в пространстве

$$
\mathscr{F}^{b}=L_{\infty}^{b}(\mathbb{R} ; H) \cap L_{2}^{b}(\mathbb{R} ; V) \cap L_{p}^{b}\left(\mathbb{R} ;\left(L_{p}(\Omega)\right)^{N}\right) \cap\left\{v: \partial_{t} v \in L_{q}^{b}\left(\mathbb{R} ;\left(H^{-r}(\Omega)\right)^{N}\right)\right\} .
$$

Множество $\mathfrak{A}_{\varepsilon}$ равномерно $($ по $\left.\left.\varepsilon \in] 0,1\right]\right)$ ограничено в $\mathscr{F}^{b}$.

Доказательство этого утверждения практически полностью совпадает с доказательством, приведенным в [15] для более частного случая.

Отметим, что $\Theta_{+}^{\text {loc }} \subset L_{2}^{\text {loc }}\left(\mathbb{R}_{+} ;\left(H^{1-\delta}(\Omega)\right)^{N}\right), 0<\delta \leqslant 1$, и, следовательно, траекторные аттракторы $\mathfrak{A}_{\varepsilon}$ и $\overline{\mathfrak{A}}$ притягивают ограниченные множества траекторий в сильной топологии пространства $L_{2}^{\text {loc }}\left(\mathbb{R}_{+} ;\left(H^{1-\delta}(\Omega)\right)^{N}\right)$.

ТЕОРема 3.5. При выполнении перечисленных выше условий

$$
\mathfrak{A}_{\varepsilon} \rightarrow \overline{\mathfrak{A}} \quad n p u \quad \varepsilon \rightarrow 0+\quad \boldsymbol{\beta} \quad \Theta_{+}^{\text {loc }}
$$

$u$

$$
\mathscr{K}_{\varepsilon} \rightarrow \overline{\mathscr{K}} \quad \text { npu } \quad \varepsilon \rightarrow 0+\quad \text { в } \quad \Theta^{\text {loc }} .
$$

ДокАЗАтЕльство. Достаточно проверить (3.33). Как и при доказательстве теоремы 3.1, предположим обратное. Тогда найдутся последовательности $\varepsilon_{n} \rightarrow 0+(n \rightarrow \infty), u_{\varepsilon_{n}}(s) \in \mathscr{K}_{\varepsilon_{n}}$ и найдется окрестность $\mathscr{O}(\overline{\mathscr{K}})$ в $\Theta^{\text {loc }}$ такие, что

$$
u_{\varepsilon_{n}}(s) \notin \mathscr{O}(\overline{\mathscr{K}}) \quad \text { для } n=1,2, \ldots .
$$

Функция $u_{\varepsilon_{n}}(s), s \in \mathbb{R}$, является решением уравнения

$$
\partial_{t} u_{\varepsilon_{n}}=a \Delta u_{\varepsilon_{n}}-b\left(x, \frac{x}{\varepsilon_{n}}\right) f\left(u_{\varepsilon_{n}}\right)+g\left(x, \frac{x}{\varepsilon_{n}}\right),\left.\quad u_{\varepsilon_{n}}\right|_{\partial \Omega}=0
$$


на всей оси времени $t \in \mathbb{R}$. Кроме того, последовательность $\left\{u_{\varepsilon_{n}}(s)\right\}$ ограничена в $\mathscr{F}^{b}$, т.е.

$$
\begin{aligned}
\left\|u_{\varepsilon_{n}}\right\|_{\mathscr{F} b}=\sup _{t \in \mathbb{R}}\left\|u_{\varepsilon_{n}}(t)\right\|_{H}+\sup _{t \in \mathbb{R}}\left(\int_{t}^{t+1}\left\|u_{\varepsilon_{n}}(s)\right\|_{V}^{2} d s\right)^{1 / 2} \\
+\sup _{t \in \mathbb{R}}\left(\int_{t}^{t+1}\left\|u_{\varepsilon_{n}}(s)\right\|_{L_{p}}^{p} d s\right)^{1 / p}+\sup _{t \in \mathbb{R}}\left(\int_{t}^{t+1}\left\|\partial_{t} u_{\varepsilon_{n}}(s)\right\|_{H^{-r}}^{q} d s\right)^{1 / q} \leqslant C \\
\forall n \in \mathbb{N} .
\end{aligned}
$$

Следовательно, найдется подпоследовательность $\left\{u_{\varepsilon_{n}^{\prime}}(s)\right\} \subset\left\{u_{\varepsilon_{n}}(s)\right\}$, которую мы вновь обозначим $\left\{u_{\varepsilon_{n}}(s)\right\}$, такая, что

$$
u_{\varepsilon_{n}}(s) \rightarrow \bar{u}(s) \text { при } n \rightarrow \infty \text { в } \Theta^{\text {loc }},
$$

где $\bar{u}(s) \in \mathscr{F}^{b}$ и $\bar{u}(s)$ удовлетворяет (3.36) с той же константой $C$. Подробнее, имеем, что $u_{\varepsilon_{n}}(s) \neg \bar{u}(s)(n \rightarrow \infty)$ слабо в $L_{2, w}^{\text {loc }}(\mathbb{R} ; V)$, слабо в $L_{p, w}^{\text {loc }}\left(\mathbb{R} ;\left(L_{p}(\Omega)\right)^{N}\right)$, *-слабо в $L_{\infty, * w}^{\text {loc }}\left(\mathbb{R}_{+} ; H\right)$ и $\partial_{t} u_{\varepsilon_{n}}(s) \rightarrow \partial_{t} \bar{u}(s)(n \rightarrow \infty)$ слабо в $L_{q, w}^{\text {loc }}\left(\mathbb{R} ;\left(H^{-r}(\Omega)\right)^{N}\right)$. Утверждается, что $\bar{u}(s) \in \overline{\mathscr{K}}$. Уже доказано, что $\|\bar{u}\|_{\mathscr{F} b} \leqslant C$. Осталось проверить, что $\bar{u}(s)$ является слабым решением (3.31). Используя (3.36) и (3.30), получаем

$$
\partial_{t} u_{\varepsilon_{n}}-a \Delta u_{\varepsilon_{n}}-g\left(x, \frac{x}{\varepsilon_{n}}\right) \rightarrow \partial_{t} \bar{u}-a \Delta \bar{u}-\bar{g}(x) \text { при } n \rightarrow \infty
$$

в пространстве $D^{\prime}\left(\mathbb{R} ;\left(H^{-r}(\Omega)\right)^{N}\right)$, потому что оператор производной непрерывен в пространстве обобщенных функций. Докажем, что

$$
b\left(x, \frac{x}{\varepsilon_{n}}\right) f\left(u_{\varepsilon_{n}}\right) \rightarrow \bar{b}(x) f(\bar{u}) \text { при } n \rightarrow \infty
$$

слабо в $L_{q, w}^{\text {loc }}\left(\mathbb{R} ;\left(L_{q}(\Omega)\right)^{N}\right)$. Зафикисируем любое число $M>0$. Последовательность $\left\{u_{\varepsilon_{n}}(s)\right\}$ ограничена в $L_{p}\left(-M, M ;\left(L_{p}(\Omega)\right)^{N}\right)$ (см. (3.36)). Тогда в силу (3.27) последовательность $\left\{f\left(u_{\varepsilon_{n}}(s)\right)\right\}$ ограничена в $L_{q}\left(-M, M ;\left(L_{q}(\Omega)\right)^{N}\right)$. Поскольку последовательность $\left\{u_{\varepsilon_{n}}(s)\right\}$ ограничена в $L_{2}\left(-M, M ;\left(H_{0}^{1}(\Omega)\right)^{N}\right)$ и $\left\{\partial_{t} u_{\varepsilon_{n}}(s)\right\}$ ограничена в $L_{q}\left(-M, M ;\left(H^{-r}(\Omega)\right)^{N}\right)$, можно предполагать, что $u_{\varepsilon_{n}}(s) \rightarrow \bar{u}(s)$ при $n \rightarrow \infty$ сильно в $L_{2}\left(-M, M ;\left(L_{2}(\Omega)\right)^{N}\right)=L_{2}(\Omega \times]-M, M[)^{N}$ и, следовательно,

$$
\left.u_{\varepsilon_{n}}(x, s) \rightarrow \bar{u}(x, s) \text { при } n \rightarrow \infty \text { для почти всех }(x, s) \in \Omega \times\right]-M, M[.
$$

Так как функция $f(v)$ непрерывна по $v \in \mathbb{R}^{N}$, заключаем

$$
\left.f\left(u_{\varepsilon_{n}}(x, s)\right) \rightarrow f(\bar{u}(x, s)) \text { при } n \rightarrow \infty \text { для почти всех }(x, s) \in \Omega \times\right]-M, M[.
$$

Имеем

$$
b\left(x, \frac{x}{\varepsilon_{n}}\right) f\left(u_{\varepsilon_{n}}\right)-\bar{b}(x) f(\bar{u})=b\left(x, \frac{x}{\varepsilon_{n}}\right)\left(f\left(u_{\varepsilon_{n}}\right)-f(\bar{u})\right)+\left(b\left(x, \frac{x}{\varepsilon_{n}}\right)-\bar{b}(x)\right) f(\bar{u}) .
$$


Покажем, что оба слагаемых в правой части (3.41) сходятся к нулю при $n \rightarrow \infty$ слабо в $L_{q}\left(-M, M ;\left(L_{q}(\Omega)\right)^{N}\right)=\left(L_{q}(\Omega \times]-M, M[)\right)^{N}$. Последовательность $b\left(x, x / \varepsilon_{n}\right)\left(f\left(u_{\varepsilon_{n}}\right)-f(\bar{u})\right)$ стремится к нулю при $n \rightarrow \infty$ для почти всех $(x, s) \in \Omega \times$ ]$-M, M\left[(\right.$ см. $(3.40))$, и она ограничена в $\left(L_{q}(\Omega \times]-M, M[)\right)^{N}($ см. $(3.28))$. Применяя лемму 1.3 из $[13 ;$ гл. $1, \S 1]$, заключаем, что

$$
b\left(x, \frac{x}{\varepsilon_{n}}\right)\left(f\left(u_{\varepsilon_{n}}\right)-f(\bar{u})\right) \rightarrow 0 \text { при } n \rightarrow \infty
$$

слабо в $\left(L_{q}(\Omega \times]-M, M[)\right)^{N}$. Последовательность $\left(b\left(x, x / \varepsilon_{n}\right)-\bar{b}(x)\right) f(\bar{u})$ также сходится к нулю при $n \rightarrow \infty$ слабо в $\left(L_{q}(\Omega \times]-M, M[)\right)^{N}$, потому что по условию $b\left(x, x / \varepsilon_{n}\right) \rightarrow \bar{b}(x)$ при $n \rightarrow \infty \quad$ *-слабо в $L_{\infty, * w}\left(-M, M ; L_{2}(\Omega)\right)$ и $f(\bar{u}) \in\left(L_{q}(\Omega \times]-M, M[)\right)^{N}$. Тем самым, (3.39) доказано. Применяя (3.38) и (3.39), делаем предельньй переход в уравнении (3.35) при $n \rightarrow \infty$ в пространстве $D^{\prime}\left(\mathbb{R}_{+} ;\left(H^{-r}(\Omega)\right)^{N}\right)$ и получаем, что функция $\bar{u}(x, s)$ является слабым решением уравнения

$$
\partial_{t} \bar{u}=a \Delta \bar{u}-\bar{b}(x) f(\bar{u})+\bar{g}(x),\left.\quad \bar{u}\right|_{\partial \Omega}=0, \quad t \in \mathbb{R} .
$$

Следовательно, $\bar{u} \in \overline{\mathscr{K}}$. Выше было доказано, что $u_{\varepsilon_{n}}(s) \rightarrow \bar{u}(s)$ при $n \rightarrow \infty$ в $\Theta^{\text {lос }}$, и из условия $u_{\varepsilon_{n}}(s) \notin \mathscr{O}(\overline{\mathscr{K}})$ следует, что $\bar{u} \notin \mathscr{O}(\overline{\mathscr{K}})$ и, тем более, $\bar{u} \notin \overline{\mathscr{K}}$. Пришли к противоречию. Теорема доказана.

СлЕДСТВИЕ 3.5. При любом $0<\delta \leqslant 1$ имеем:

$$
\operatorname{dist}_{L_{2}\left(0, M ; H^{1-\delta}\right)}\left(\Pi_{0, M} \mathfrak{A}_{\varepsilon}, \Pi_{0, M} \overline{\mathfrak{A}}\right) \rightarrow 0 \quad(\varepsilon \rightarrow 0+) \quad \forall M>0
$$

ЗАмечАниЕ 3.3. Аналогичная теорема справедлива для неавтономных систем реакции-диффузии вида (3.26), которые содержат члены $b(x, t / \varepsilon, t)$ и $g(x, t / \varepsilon, t)$, имеющие равномерные средние при $\varepsilon \rightarrow 0+$.

Применим теорему 3.5 для усреднения комплексного уравнения Гинзбурга-Ландау вида

$$
\partial_{t} u=(1+\alpha i) \Delta u+R\left(\frac{x}{\varepsilon}\right) u+\left(1+\beta\left(\frac{x}{\varepsilon}\right) i\right)|u|^{2} u+g\left(\frac{x}{\varepsilon}\right)
$$

с периодическими граничными условиями. Здесь $x \in[0,2 \pi]^{n}, u=u_{1}+i u_{2} \in \mathbb{C}$, $g(z) \in L_{2}^{\text {loc }}\left(\mathbb{R}^{N} ; \mathbb{C}\right)$ и $R(z), \beta(z) \in C_{b}\left(\mathbb{R}^{N}\right)$. Предполагается, что

$$
0<R_{1} \leqslant R(z) \leqslant R_{2}, \quad 0<\beta_{1} \leqslant \beta(z) \leqslant \beta_{2} \quad \text { при } z \in \mathbb{R}^{N},
$$

а функции $R(x / \varepsilon)$ и $\beta(x / \varepsilon)$ имеют постоянные средние $\bar{R}$ и $\bar{\beta}$ в $L_{\infty, * w}\left([0,2 \pi]^{n}\right)$ соответственно. Пусть также функция $g(x / \varepsilon)$ имеет среднее $\bar{g}$ в $L_{2, w}^{\text {loc }}\left([0,2 \pi]^{n} ; \mathbb{C}\right)$. Легко проверить, что функция $f(v)=(1+\beta(z) i)|v|^{2} v$ удовлетворяет (3.27) со степенью $p=4$.

Усредненное уравнение для (3.42) имеет вид

$$
\partial_{t} \bar{u}=(1+\alpha i) \Delta \bar{u}+\bar{R} \bar{u}+(1+\bar{\beta} i)|\bar{u}|^{2} \bar{u}+\bar{g} .
$$

Уравнения (3.42) и (3.43) имеют траекторные аттракторы $\mathfrak{A}_{\varepsilon}$ и $\overline{\mathfrak{A}}$ соответственно в пространстве $\Theta_{+}^{\text {loc }}$, отвечающем этим уравнениям. Из теоремы 3.5 следует, что 
траекторный аттрактор $\mathfrak{A}_{\varepsilon}$ уравнения $(3.42)$ стремится при $\varepsilon \rightarrow 0+$ в $\Theta_{+}^{\text {loc }}$ в указанном вьше смысле к траекторному аттрактору $\overline{\mathfrak{A}}$ усредненного уравнения (3.43).

В заключение рассмотрим системы реакции-диффузии, для которых имеет место теорема единственности соответствуюшей задачи Коши. Для этого достаточно, чтобы нелинейная вектор-функция $f(u)$ в уравнении (3.26) удовлетворяла условию:

$$
\left(f\left(v_{1}\right)-f\left(v_{2}\right), v_{1}-v_{2}\right) \geqslant-C\left|v_{1}-v_{2}\right|^{2} \text { при } v_{1}, v_{2} \in \mathbb{R}^{N}
$$

(см. [15]). Например, можно доказать, что уравнение Гинзбурга-Ландау (3.42) удовлетворяет (3.44), если $|\beta| \leqslant \sqrt{3}$.

Легко устанавливается, что при выполнении условия (3.44) предел (3.32) имеет место в более сильном пространстве

$$
\begin{gathered}
\Theta_{+}^{\mathrm{loc}, 1}=L_{\infty, * w}^{\mathrm{loc}}\left(\mathbb{R}_{+} ;\left(H_{0}^{1}(\Omega)\right)^{N}\right) \cap L_{2, w}^{\mathrm{loc}}\left(\mathbb{R}_{+} ;\left(H^{2}(\Omega)\right)^{N}\right) \cap L_{p, w}^{\mathrm{loc}}\left(\mathbb{R}_{+} ;\left(L_{p}(\Omega)\right)^{N}\right) \\
\cap\left\{v: \partial_{t} v \in L_{q, w}^{\mathrm{loc}}\left(\mathbb{R}_{+} ;\left(L_{q}(\Omega)\right)^{N}\right)\right\} .
\end{gathered}
$$

В частности,

$$
\begin{aligned}
& \operatorname{dist}_{L_{2}\left(0, M ; H^{2-\delta}\right)}\left(\Pi_{0, M} \mathfrak{A}_{\varepsilon}, \Pi_{0, M} \overline{\mathfrak{A}}\right) \rightarrow 0 \quad(\varepsilon \rightarrow 0+) \\
& \operatorname{dist}_{C\left([0, M] ; H^{1-\delta}\right)}\left(\Pi_{0, M} \mathfrak{A}_{\varepsilon}, \Pi_{0, M} \overline{\mathfrak{A}}\right) \rightarrow 0 \quad(\varepsilon \rightarrow 0+) \quad \forall M>0, \quad 0<\delta \leqslant 1 .
\end{aligned}
$$

В работе [15] доказано, что при выполнении условия (3.44) уравнения (3.26) и (3.31) порождают полугруппы $\left\{S_{\varepsilon}(t)\right\}$ и $\{\bar{S}(t)\}$ в $H=\left(L_{2}(\Omega)\right)^{N}$, которые имеют глобальные аттракторы $\mathscr{A}_{\varepsilon}$ и $\overline{\mathscr{A}}$, ограниченные в пространстве $\left(H_{0}^{1}(\Omega)\right)^{N}$ (см. также [7], [8]). В силу теоремы 2.4 получаем:

$$
\mathscr{A}_{\varepsilon}=\left\{u(0): u \in \mathfrak{A}_{\varepsilon}\right\}, \quad \overline{\mathscr{A}}=\{u(0): u \in \overline{\mathfrak{A}}\} .
$$

Из соотношения (3.45) вытекает

СлЕДСТВИЕ 3.6. Имеет место предел:

$$
\left.\left.\operatorname{dist}_{H^{1-\delta}}\left(\mathscr{A}_{\varepsilon}, \overline{\mathscr{A}}\right) \rightarrow 0 \quad(\varepsilon \rightarrow 0+) \quad \forall \delta \in\right] 0,1\right]
$$

В конкретных примерах можно доказать более сильные результаты о сходимости траекторных аттракторов $\mathfrak{A}_{\varepsilon}$ и глобальных аттракторов $\mathscr{A}_{\varepsilon}$ к аттракторам усредненных уравнений. В следующем параграфе изучаются такие примеры.

3.4. Обобщенное уравнение Чэфи-Инфанте. Изучается следующее обобщение уравнения Чэфи-Инфанте (см. [16], [17]) на $n$-мерный случай:

$$
\partial_{t} u=\Delta u-\sum_{j=0}^{N} b_{j}\left(\frac{x}{\varepsilon}\right) u^{j} \equiv \Delta u-f\left(u, \frac{x}{\varepsilon}\right),\left.\quad u\right|_{\partial \Omega}=0,
$$

где $x \in \Omega \Subset \mathbb{R}^{n}, N=2 l+1$. Предполагается, что $b_{j}(z) \in C_{b}\left(\mathbb{R}^{n}\right)\left(z=\left(z_{1}, \ldots, z_{n}\right)\right)$ и

$$
b_{N}(z) \geqslant \beta_{N}>0 \quad \forall z \in \mathbb{R}^{n} .
$$

Как обычно, предположим, что

$$
b_{j}\left(\frac{x}{\varepsilon}\right) \rightarrow \bar{b}_{j} \quad \text { в } \quad L_{\infty, * w}(\Omega) \quad(j=0, \ldots, N) .
$$

Усредненное уравнение примет вид:

$$
\partial_{t} \bar{u}=\Delta \bar{u}-\sum_{j=0}^{N} \bar{b}_{j} \bar{u}^{j} \equiv \Delta \bar{u}-\bar{f}(\bar{u}),\left.\quad \bar{u}\right|_{\partial \Omega}=0 .
$$


ЗАмечАниЕ 3.4. Аналогичные результаты можно распространить на случай более общих уравнений и систем. Например, в (3.47) можно считать, что $f(v, z) \in$ $C\left(\mathbb{R} \times R^{n} ; \mathbb{R}\right)$ и выполнены неравенства:

$$
\begin{aligned}
& f(v, z) \cdot v \geqslant-C+\gamma|v|^{p}, \quad p>0, \\
& |f(v, z)| \leqslant C_{1}\left(|v|^{p-1}+1\right) \quad \forall v \in \mathbb{R}, \quad z \in \mathbb{R}^{n} .
\end{aligned}
$$

Ясно, что функция $f(v, z)=\sum_{j=0}^{N} b_{j}(z) v^{j}$ удовлетворяет (3.51) и (3.52) при $p=2(l+1)$.

Как и раншше, обозначим через $\mathscr{K}_{\varepsilon}^{+}, \varepsilon>0$, множество слабых решений уравнения $(3.47)$ из пространства $\mathscr{F}_{+}^{b}=L_{\infty}^{b}\left(\mathbb{R}_{+} ; L_{2}(\Omega)\right) \cap L_{2}^{b}\left(\mathbb{R}_{+} ; H_{0}^{1}(\Omega)\right) \cap L_{p}^{b}\left(\mathbb{R}_{+} ; L_{p}(\Omega)\right)$. Аналогично обозначаем через $\overline{\mathscr{K}}^{+}$пространство траекторий для (3.50). Уравнения (3.47) и (3.50) имеют траекторные аттракторы $\mathfrak{A}_{\varepsilon}$ и $\overline{\mathfrak{A}}$, которые ограничены в $\mathscr{F}_{+}^{b}$ и компактны в пространстве $\Theta_{+}^{\text {loc }}$, определенном в предыдущем параграфе. Там же доказано, что $\mathfrak{A}_{\varepsilon} \rightarrow \overline{\mathfrak{A}}_{\text {при }} \varepsilon \rightarrow 0+$ в $\Theta_{+}^{\text {loc }}$. Теперь мы докажем, что траекторный аттрактор $\mathfrak{A}_{\varepsilon}$ уравнения (3.47) сходится к траекторному аттрактору $\overline{\mathfrak{A}}$ уравнения $(3.50)$ при $\varepsilon \rightarrow 0+$ в пространстве $C_{\text {loc }}^{2-2 \delta, 1-\delta}\left(\Omega \times \mathbb{R}_{+}\right), 1>\delta>0$.

УТВЕРЖДЕНИЕ 3.4. Траекторные аттракторы $\mathfrak{A}_{\varepsilon}$ и $\overline{\mathfrak{A}}$ ограничены в пространстве $L_{\infty}\left(\mathbb{R} ; L_{\infty}(\Omega)\right)=L_{\infty}(\Omega \times \mathbb{R})$ равномерно по $\varepsilon>0$.

Доказательство см. [8; гл. IV, утверждение 6.2].

Нам понадобятся некоторые дополнительные определения и обозначения. Обозначим через $\left.Q_{T}=\Omega \times\right] T, T+1[, T \in \mathbb{R}$, цилиндр с высотой 1 . Будем рассматривать слабые решения $u(x, t)$ уравнений $(3.47)$ и (3.50) такие, что

$$
u(x, s) \in W_{p}^{\mathbf{r}}\left(Q_{T}\right) \cap\left\{\left.u\right|_{\partial \Omega}=0\right\} \equiv W_{p, 0}^{\mathbf{r}}\left(Q_{T}\right) \quad \forall T \in \mathbb{R},
$$

где $\mathbf{r}=(2, \ldots, 2,1)$ и $W_{p}^{\mathbf{r}}\left(Q_{T}\right)$ обозначает пространство Никольского. Напомним, что норма в $W_{p}^{\mathbf{r}}\left(Q_{T}\right)$ задается выражением

$$
\left\|u, Q_{T}\right\|_{\mathbf{r}, p}^{p}=\int_{Q_{T}}\left(\sum_{|\alpha| \leqslant 2}\left|\partial_{x}^{\alpha} u(x, s)\right|^{p}+\left|\partial_{t} u(x, s)\right|^{p}\right) d x d s .
$$

Теорема вложения Никольского утверждает (см. [18]), что если $p>(n+2) / 2$, то

$$
W_{p, 0}^{\mathbf{r}}\left(Q_{T}\right) \Subset C^{\varkappa \mathbf{r}}\left(\bar{Q}_{T}\right) \cap\left\{\left.u\right|_{\partial \Omega}=0\right\} \equiv C_{0}^{\varkappa \mathbf{r}}\left(\bar{Q}_{T}\right),
$$

где

$$
0<\varkappa<1-\frac{1}{p}\left(1+\frac{n}{2}\right) .
$$

Следовательно, для фиксированного $\delta, 0<\delta<1$, из (3.53) следует, что при $\varkappa=1-\delta$ имеет место компактное вложение:

$$
W_{p, 0}^{\mathbf{r}}\left(Q_{T}\right) \Subset C_{0}^{2-2 \delta, 1-\delta}\left(\bar{Q}_{T}\right) \quad \text { для } p>\frac{n+2}{2 \delta} .
$$

Нам потребуется следующая теорема Солонникова (см. [19]). Обозначим через $V_{0}$ пространство следов функций из $\left.W_{p, 0}^{\mathbf{r}}\left(Q_{0}\right), Q_{0}=\Omega \times\right] 0,1[$, на сечениях 
$\{t=0\}$. Пусть функция $w(x, t) \in L_{\infty}\left(0,1 ; L_{\infty}(\Omega)\right) \cap L_{2}\left(0,1 ; H_{0}^{1}(\Omega)\right)$ является решением уравнения теплопроводности

$$
\partial_{t} w-\Delta w=h(x, t), \quad(x, t) \in Q_{0},
$$

и удовлетворяет начальному условию

$$
\left.w\right|_{t=0}=w_{0}(x) .
$$

Предположим, что $h(x, t) \in L_{p}\left(0,1 ; L_{p}(\Omega)\right) \equiv L_{p}\left(Q_{0}\right)$ и $w_{0} \in V_{0}$. Тогда $w(x, t) \in$ $W_{p, 0}^{\mathrm{r}}\left(Q_{0}\right)$ и

$$
\left\|w, Q_{0}\right\|_{\mathbf{r}, p} \leqslant C_{p}\left(\|h\|_{L_{p}\left(Q_{0}\right)}+\left\|w_{0}\right\|_{V_{0}}\right) .
$$

Обозначим через $\mathscr{W}_{p}^{\text {loc }}$ пространство функций $v(x, s)$ таких, что

$$
\Pi_{T, T+1} v(x, s) \in W_{p, 0}^{\mathbf{r}}\left(Q_{T}\right) \quad \forall T \in \mathbb{R} .
$$

Как обычно, определяется пространство $\mathscr{W}_{p}^{b} \subset \mathscr{W}_{p}^{\text {loc }}$ с нормой

$$
\|v\|_{\mathscr{W}_{p}^{b}}=\sup _{T \in \mathbb{R}}\left\|\Pi_{T, T+1} v, Q_{T}\right\|_{\mathbf{r}, p}
$$

Наконец, $\mathscr{W}_{p, w}^{\text {loc }}$ обозначает пространство $\mathscr{W}_{p}^{\text {loc }}$ с топологией локальной слабой сходимости $W_{p, 0}^{\mathbf{r}}\left(Q_{T}\right)$ при любом $T \in \mathbb{R}$. Из (3.54) заключаем, что

$$
\Theta^{\mathrm{loc}} \Subset C_{0, \mathrm{loc}}^{2-2 \delta, 1-\delta}(\bar{\Omega} \times \mathbb{R}) \text { для } p>\frac{n+2}{2 \delta} .
$$

Имеет место следующая

Tеорема 3.6. Траекторный аттрактор $\mathfrak{A}_{\varepsilon}$ уравнения (3.47) равномерно (по $\varepsilon>0$ ) ограничен в $\mathscr{W}_{p}^{b}$ и $\mathfrak{A}_{\varepsilon}$ стремится $\kappa$ траекторному аттрактору $\overline{\mathfrak{A}}$ уравнения (3.50) при $\varepsilon \rightarrow 0+$ в пространстве $\mathscr{W}_{p, w}^{\text {loc }}$. В частности, из вложения (3.58) следует, что

$$
\operatorname{dist}_{C^{2-2 \delta, 1-\delta}(\bar{\Omega} \times[0, M])}\left(\Pi_{M} \mathfrak{A}_{\varepsilon}, \Pi_{M} \overline{\mathfrak{A}}\right) \rightarrow 0 \quad(\varepsilon \rightarrow 0+) \quad \forall M>0 .
$$

ДоКАЗАТЕЛЬСтво. Пусть $u \in \mathscr{K}_{\varepsilon}$ и $\bar{u} \in \overline{\mathscr{K}}$. Из утверждения 3.4 и из неравенства (3.52) вытекает, что правые части уравнений

$$
\begin{aligned}
& \partial_{t} u-\Delta u=-f\left(u, \frac{x}{\varepsilon}\right),\left.\quad u\right|_{\partial \Omega}=0, \quad 0<\varepsilon \leqslant 1, \\
& \partial_{t} \bar{u}-\Delta \bar{u}=-\bar{f}(\bar{u}),\left.\quad \bar{u}\right|_{\partial \Omega}=0
\end{aligned}
$$

ограничены в $L_{\infty}(\Omega \times \mathbb{R})$ равномерно по $\varepsilon>0$. Поэтому ядра $\mathscr{K}_{\varepsilon}$ и $\overline{\mathscr{K}}$ принадлежат множеству

$$
B_{0}=\left\{u:\|u\|_{\mathscr{W}_{p}^{b}} \leqslant R_{0}\right\}
$$


где $R_{0}$ не зависит от $\varepsilon$. В самом деле, умножим уравнение (3.60) на срезаюшую монотонную функцию $\Phi_{T}(t)=\Phi(t-T)$, где $\Phi(s) \in C_{b}^{1}\left(\mathbb{R} ; \mathbb{R}_{+}\right), \Phi(s)=0$ при $s \leqslant-1$ и $\Phi(s)=1$ при $s \geqslant 0$. Пусть $T \geqslant 1$. Получаем:

$$
\partial_{t}\left(\Phi_{T} u\right)-\Delta\left(\Phi_{T} u\right)=-\Phi_{T} f\left(u, \frac{x}{\varepsilon}\right)-\Phi_{T}^{\prime} u
$$

Поскольку $\Phi_{T}(t) u(x, t) \equiv 0$ при $t=T-1$, можно применить оценку Солонникова $(3.55)$ в области $\left.Q_{T-1, T+1}=\Omega \times\right] T-1, T+1[$ и получить неравенство

$$
\begin{aligned}
\left\|u, Q_{T}\right\|_{\mathbf{r}, p} & \leqslant\left\|\Phi_{T} u, Q_{T-1, T+1}\right\|_{\mathbf{r}, p} \\
& \leqslant C_{p}\left\|-\Phi_{T} f\left(u, \frac{x}{\varepsilon}\right)-\Phi_{T}^{\prime} u\right\|_{L_{p}\left(Q_{T-1, T+1}\right)} \leqslant R_{0} \quad \forall T \in \mathbb{R} .
\end{aligned}
$$

Мы доказали, что множество $B_{0}$ (см. (3.62)) содержит $\mathscr{K}_{\varepsilon}$ и $\overline{\mathscr{K}}$.

В силу того, что множество $B_{0}$ компактно в $\mathscr{W}_{p, w}^{\text {loc }}$, получаем, используя стандартные рассуждения, что $\mathscr{K}_{\varepsilon} \rightarrow \overline{\mathscr{K}}$ при $\varepsilon \rightarrow 0+$ в $W_{p, w}^{\mathbf{r}}(\Omega \times]-M, M[)$ и, следовательно, $\mathfrak{A}_{\varepsilon} \rightarrow \overline{\mathfrak{A}}_{\text {при }} \varepsilon \rightarrow 0+$ в $W_{p, w}^{\mathbf{r}}(\Omega \times] 0, M[)$ для любого $M>0$. В частности, имеет место (3.59).

Теорема 3.6 влечет за собою

СледСтвиЕ 3.7. Глобальные аттракторы $\mathscr{A}_{\varepsilon}$ и $\overline{\mathscr{A}}$ уравнений (3.47) и (3.50) принадлежсат $C^{2-\delta}(\bar{\Omega})$ u

$$
\left.\left.\operatorname{dist}_{C^{2-\delta}(\bar{\Omega})}\left(\mathscr{A}_{\varepsilon}, \overline{\mathscr{A}}\right) \rightarrow 0 \quad(\varepsilon \rightarrow 0+) \quad \forall \delta \in\right] 0,1\right]
$$

ЗАмЕчАниЕ 3.5. Аналогичными методами исследуются равномерные траекторные и глобальные аттракторы неавтономных уравнений вида $(3.26)$ с нелинейными функциями взаимодействия $f(v, x, x / \varepsilon, t, t / \varepsilon)=\sum_{j=0}^{N} b_{j}(x, x / \varepsilon, t, t / \varepsilon) v^{j}$, где коэффициенты $b_{j}\left(x, z_{1}, t, z_{2}\right)$ имеют средние в соответствующих пространствах.

3.5. Диссипативное волновое уравнение. Рассматривается гиперболическое уравнение с диссипацией:

$$
\partial_{t}^{2} u+\gamma\left(x, \frac{x}{\varepsilon}\right) \partial_{t} u=\Delta u-b\left(x, \frac{x}{\varepsilon}\right) f(u)+g\left(x, \frac{x}{\varepsilon}\right),\left.\quad u\right|_{\partial \Omega}=0 .
$$

Здесь $x \in \Omega \Subset \mathbb{R}^{n}$. Аналогичное гиперболическое уравнение с постоянными коэффициентами $\gamma$ и $b$ изучалось в [6]. Изложенный там метод построения траекторного аттрактора вполне применим к уравнению (3.65) без существенных изменений.

Предполагается, что $\gamma(x, z), b(x, z) \in C_{b}(\bar{\Omega} \times \mathbb{R})$ и

$$
\begin{aligned}
& 0<\bar{\gamma}_{1} \leqslant \gamma(x, z) \leqslant \bar{\gamma}_{2}, \\
& 0<\bar{\beta}_{1} \leqslant b(x, z) \leqslant \bar{\beta}_{2} \quad \forall x \in \bar{\Omega} \times \mathbb{R}^{n} .
\end{aligned}
$$

Функция $f(v) \in C(\mathbb{R})$ удовлетворяет неравенствам

$$
\begin{array}{ll}
|f(v)| \leqslant \gamma_{0}\left(|v|^{p-1}+1\right), & p>2, \\
F(v) \geqslant \gamma_{3}|v|^{p}-C_{1}, \quad & f(v) v \geqslant \gamma_{4} F(v)-C_{2} \quad \forall v \in \mathbb{R},
\end{array}
$$


где $F(v)=\int_{0}^{v} f(w) d w$ и функция $\Phi(v)=\left(f(v)+C_{1}\right)^{1 / p}$ удовлетворяет условию Липшица (см. [6]).

Пусть функции $\gamma(x, x / \varepsilon)$ и $b(x, x / \varepsilon)$ имеют средние $\bar{\gamma}(x)$ и $\bar{b}(x)$ при $\varepsilon \rightarrow 0+$ в пространстве $L_{\infty, * w}(\Omega)$, т.е.

$$
\begin{aligned}
& \left\langle\gamma\left(x, \frac{x}{\varepsilon}\right), \varphi(x)\right\rangle \rightarrow\langle\bar{\gamma}(x), \varphi(x)\rangle, \\
& \left\langle b\left(x, \frac{x}{\varepsilon}\right), \varphi(x)\right\rangle \rightarrow\langle\bar{b}(x), \varphi(x)\rangle \quad(\varepsilon \rightarrow 0+)
\end{aligned}
$$

для любой функции $\varphi \in L_{1}(\Omega)$. Наконец, предположим, что функция $g(x, x / \varepsilon)$ имеет среднее $\bar{g}(x)$ при $\varepsilon \rightarrow 0+$ в $L_{2, w}(\Omega)$ :

$$
\left\langle g\left(x, \frac{x}{\varepsilon}\right), \varphi(x)\right\rangle \rightarrow\langle\bar{g}(x), \varphi(x)\rangle \quad(\varepsilon \rightarrow 0+) \forall \varphi \in L_{2}(\Omega) .
$$

ЗАмЕчАнИЕ 3.6. Излагаемые далее результаты легко обобшаются на случай, когда $g=g(x, x / \varepsilon, t, t / \varepsilon), \gamma=\gamma(x, x / \varepsilon, t, t / \varepsilon)$. Можно также рассматривать нелинейные члены вида $\sum_{j=1}^{N} \beta_{j}\left(x, \frac{x}{\varepsilon}, t, \frac{t}{\varepsilon}\right) f_{j}(v)$ u ux предель.

Рассмотрим также усредненное уравнение

$$
\partial_{t}^{2} \bar{u}+\bar{\gamma}(x) \partial_{t} \bar{u}=\Delta \bar{u}-\bar{b}(x) f(\bar{u})+\bar{g}(x),\left.\quad \bar{u}\right|_{\partial \Omega}=0 .
$$

При выполнении условий (3.66)-(3.71) уравнение (3.65) при $\varepsilon>0$ и уравнение (3.72) имеют траекторные аттракторы $\mathfrak{A}_{\varepsilon}$ и $\overline{\mathfrak{A}}$ соответственно. Конструкция и свойства этих аттракторов описаны в [6].

Напомним, что функция $u(x, s)$ называется слабым решением уравнения $(3.65)$ (или (3.72)), если $u(x, s) \in L_{\infty}^{\text {loc }}\left(\mathbb{R}_{+} ; L_{p}(\Omega) \cap H_{0}^{1}(\Omega)\right), \partial_{t} u \in L_{\infty}^{\text {loc }}\left(\mathbb{R}_{+} ; L_{2}(\Omega)\right.$ ) и $u$ удовлетворяет уравнению в смысле обобшенных функций пространства $D^{\prime}\left(\mathbb{R}_{+} ; H^{-r}(\Omega)\right)$, где $r=\max \{1 ; n(1 / 2-1 / p)\}$. Тогда очевидно, что $\partial_{t}^{2} u \in$ $L_{\infty}^{\text {loc }}\left(\mathbb{R}_{+} ; H^{-r}(\Omega)\right)$.

Аналогично [6] вводим функционал

$$
J_{\varepsilon, \alpha}\left(v, v_{1}\right)=\int_{\Omega}\left(|\nabla v(x)|^{2}+\left|v_{1}(x)+\alpha v(x)\right|^{2}+2 b\left(x, \frac{x}{\varepsilon}\right) F(v(x))\right) d x .
$$

Пусть $u(x, s)$ - слабое решение (3.65). Обозначим

$$
z(s)=J_{\varepsilon, \alpha}\left(u(s), \partial_{t} u(s)\right) .
$$

Зафиксируем число $N>0$. Пространство траекторий $\mathscr{K}_{\varepsilon}^{+}(N)$ уравнения (3.65) состоит из слабых решений этого уравнения, которые удовлетворяют неравенству

$$
z(t) \leqslant R_{\alpha}+N \exp \left(-\delta_{\alpha} t\right) \quad \forall t \geqslant 0
$$

для любого $\alpha$ такого, что

$$
0<\alpha\left(\bar{\gamma}_{1}-\alpha\right)<\lambda_{1}, \quad 0<\alpha<\bar{\gamma}_{1} .
$$


Константы $R_{\alpha}$ и $\delta_{\alpha}$ определены в [6]. Они не зависят от $\varepsilon$. Аналогично определяется траекторное пространство $\overline{\mathscr{K}}^{+}(N)$ для уравнения (3.72). Полугруппа сдвигов $\{T(t)\}$, действующая на $\mathscr{K}_{\varepsilon}^{+}(N)$ и $\overline{\mathscr{K}}^{+}(N)$ соответственно, имеет траекторные аттракторы $\mathfrak{A}_{\varepsilon}$ и $\overline{\mathfrak{A}}$. Пространства $\Theta_{+}^{\text {loc }}$ и $\mathscr{F}_{+}^{b}$ определяются следуюшим образом:

$$
\begin{aligned}
& \Theta_{+}^{\mathrm{loc}}=L_{\infty, * w}^{\mathrm{loc}}\left(\mathbb{R}_{+} ; L_{p}(\Omega) \cap H_{0}^{1}(\Omega)\right) \cap\left\{v: \partial_{t} v \in L_{\infty, * w}^{\mathrm{loc}}\left(\mathbb{R}_{+} ; L_{2}(\Omega)\right)\right\} \cap\left\{v: \partial_{t}^{2} v \in L_{\infty, * w}^{\mathrm{loc}}\left(\mathbb{R}_{+} ; H^{-r}(\Omega)\right)\right\}, \\
& \mathscr{F}_{+}^{b}=L_{\infty}^{b}\left(\mathbb{R}_{+} ; L_{p}(\Omega) \cap H_{0}^{1}(\Omega)\right) \cap\left\{v: \partial_{t} v \in L_{\infty}^{b}\left(\mathbb{R}_{+} ; L_{2}(\Omega)\right)\right\} \\
& \cap\left\{v: \partial_{t}^{2} v \in L_{\infty}^{b}\left(\mathbb{R}_{+} ; H^{-r}(\Omega)\right)\right\} .
\end{aligned}
$$

Множества $\mathfrak{A}_{\varepsilon}$ и $\overline{\mathfrak{A}}$ равномерно ограничены в $\mathscr{F}_{+}^{b}$. Доказательство этих утверждений изложено в [6]. Там же установлено, что

$$
\mathfrak{A}_{\varepsilon}=\Pi_{+} \mathscr{K}_{\varepsilon} \quad \text { и } \overline{\mathfrak{A}}=\Pi_{+} \overline{\mathscr{K}},
$$

где $\mathscr{K}_{\varepsilon}$ и $\overline{\mathscr{K}}$ - ядра уравнений (3.65) и (3.72) соответственно. Легко видеть из (3.73), что $\mathscr{K}_{\varepsilon}$ состоит из всех слабых решений $u(x, s), s \in \mathbb{R}$, уравнения (3.65) таких, что

$$
z(t)=J_{\varepsilon, \alpha}\left(u(t), \partial_{t} u(t)\right) \leqslant R_{\alpha} \quad \forall t \in \mathbb{R} .
$$

Аналогично, ядро $\overline{\mathscr{K}}$ уравнения (3.72) состоит из его слабых решений $\bar{u}(x, s)$, удовлетворяющих неравенству

$$
\bar{z}(t)=\bar{J}_{\alpha}\left(\bar{u}(t), \partial_{t} \bar{u}(t)\right) \leqslant R_{\alpha} \quad \forall t \in \mathbb{R},
$$

где при определении $\bar{J}_{\alpha}\left(v, v_{1}\right)$ необходимо заменить $b(x, x / \varepsilon)$ в $(3.73)$ на $\bar{b}(x)$. Отметим, что траекторные аттракторы $\mathfrak{A}_{\varepsilon}$ и $\overline{\mathfrak{A}}$ не зависят от константы $N$ из определения пространств $\mathscr{K}_{\varepsilon}^{+}(N)$ и $\overline{\mathscr{K}}^{+}(N)$.

ТеОРема 3.7. Траекторный аттрактор $\mathfrak{A}_{\varepsilon}=\Pi_{+} \mathscr{K}_{\varepsilon}$ уравнения (3.65) стремится $\kappa$ траекторному аттрактору $\overline{\mathfrak{A}}=\Pi_{+} \overline{\mathscr{K}}$ уравнения $(3.72)$ при $\varepsilon \rightarrow 0+$ в пространстве $\Theta_{+}^{\text {loc }}$,

$$
\mathfrak{A}_{\varepsilon} \rightarrow \overline{\mathfrak{A}} \quad \text { npu } \quad \varepsilon \rightarrow 0+
$$

$u$

$$
\mathscr{K}_{\varepsilon} \rightarrow \overline{\mathscr{K}} \quad \text { npu } \quad \varepsilon \rightarrow 0+\quad \boldsymbol{\theta} \quad \Theta^{\mathrm{loc}} .
$$

СледСтвиЕ 3.8. В силу вложения $\Theta_{+}^{\text {loc }} \subset C\left(\mathbb{R}_{+} ; H^{1-\delta}\right) \cap C^{1}\left(\mathbb{R}_{+} ; H^{-\delta}\right)$ nри $\delta \in] 0,1]$ имеем

$$
\operatorname{dist}_{C\left([0, M] ; H^{1-\delta}\right) \cap C^{1}\left([0, M] ; H^{-\delta}\right)}\left(\Pi_{0, M} \mathfrak{A}_{\varepsilon}, \Pi_{0, M} \overline{\mathfrak{A}}\right) \rightarrow 0 \quad(\varepsilon \rightarrow 0+) \forall M>0 .
$$

Мы не приводим доказательства теоремы 3.7. Оно во многом аналогично доказательству теорем 3.1 и 3.5 . 
3.6. Гиперболическое уравнение с единственностью решения задачи Коши. В этом пункте мы изучим усреднение диссипативных гиперболических уравнений с умеренным ростом нелинейной функции. Это условие позволяет доказать однозначную разрешимость соответствуюшей задачи Коши (см. [13], [7], [8]).

Рассматривается уравнение

$$
\partial_{t}^{2} u+\gamma \partial_{t} u=\Delta u-b\left(x, \frac{x}{\varepsilon}\right) f(u)+g\left(x, \frac{x}{\varepsilon}\right),\left.\quad u\right|_{\partial \Omega}=0, \quad \varepsilon>0
$$

Здесь, как обычно, $x \in \Omega \Subset \mathbb{R}^{n}, \gamma>0$, а функция $f(v) \in C^{1}(\mathbb{R})$ удовлетворяет (3.68) и (3.69). Кроме того, предполагается, что

$$
\left|f^{\prime}(v)\right| \leqslant C\left(1+|v|^{p-2}\right), \quad \text { где } \begin{cases}2 \leqslant p<+\infty & \text { при } n=1,2 ; \\ 2 \leqslant p<4 & \text { при } n=3 ; \\ p=2 & \text { при } n \geqslant 4 .\end{cases}
$$

Пусть также $b(x, z) \in C_{b}(\bar{\Omega} \times \mathbb{R}), 0<\beta_{1} \leqslant b(x, z) \leqslant \beta_{2}$, и $b(x, x / \varepsilon)$ имеет среднее $\bar{b}(x)$ при $\varepsilon \rightarrow 0+$ в $L_{\infty, * w}(\Omega)$. Функция $g(x, x / \varepsilon) \in L_{2}(\Omega)$ при любом $\varepsilon>0$, и она имеет среднее $\bar{g}(x)$ при $\varepsilon \rightarrow 0+$ в $L_{2, w}(\Omega)$.

Рассмотрим усредненное уравнение

$$
\partial_{t}^{2} \bar{u}+\gamma \partial_{t} \bar{u}=\Delta \bar{u}-\bar{b}(x) f(\bar{u})+\bar{g}(x),\left.\quad \bar{u}\right|_{\partial \Omega}=0 .
$$

Начальные условия задаются при $t=0$ :

$$
\left.u\right|_{t=0}=u_{0}(x) \in H^{1},\left.\quad \partial_{t} u\right|_{t=0}=u_{1}(x) \in H
$$

где обозначены пространства $H=L_{2}(\Omega)$ и $H^{1}=H_{0}^{1}(\Omega)$.

Известно, что задачи $(3.80),(3.83)$ и $(3.82),(3.83)$ (с заменой $u$ на $\bar{u}$ ) имеют единственные решения $u(t), t \geqslant 0$, и $\bar{u}(t), t \geqslant 0$, такие, что $u, \bar{u} \in L_{\infty}\left(\mathbb{R}_{+} ; H^{1}\right)$, $\partial_{t} u, \partial_{t} \bar{u} \in L_{\infty}\left(\mathbb{R}_{+} ; H\right)$. Тогда из уравнений получаем, что $\partial_{t}^{2} u, \partial_{t}^{2} \bar{u} \in L_{\infty}\left(\mathbb{R}_{+} ; H^{-1}\right)$, где $H^{-1}=H^{-1}(\Omega)$ (см. [13], [8], [7]). Присутствие членов $b(x, x / \varepsilon)$ и $\bar{b}(x)$, зависящих от $x$, не влияет на эти факты.

Теперь определим пространства $\mathscr{F}_{+}^{\text {loc }}$ и $\mathscr{F}_{+}^{b}$ следующим образом:

$$
\begin{aligned}
\mathscr{F}_{+}^{\text {loc }} & =\left\{v(s), s \geqslant 0: v \in L_{\infty}^{\text {loc }}\left(\mathbb{R}_{+} ; H^{1}\right), \partial_{t} v \in L_{\infty}^{\text {loc }}\left(\mathbb{R}_{+} ; H\right), \partial_{t}^{2} v \in L_{\infty}^{\text {loc }}\left(\mathbb{R}_{+} ; H^{-1}\right)\right\} \\
\mathscr{F}_{+}^{b} & =\left\{v(s), s \geqslant 0: v \in L_{\infty}\left(\mathbb{R}_{+} ; H^{1}\right), \partial_{t} v \in L_{\infty}\left(\mathbb{R}_{+} ; H\right), \partial_{t}^{2} v \in L_{\infty}\left(\mathbb{R}_{+} ; H^{-1}\right)\right\} .
\end{aligned}
$$

Норма в пространстве $\mathscr{F}_{+}^{b}$

$$
\|v\|_{\mathscr{F}_{+}^{b}}=\|v\|_{L_{\infty}\left(\mathbb{R}_{+} ; H^{1}\right)}+\left\|\partial_{t} v\right\|_{L_{\infty}\left(\mathbb{R}_{+} ; H\right)}+\left\|\partial_{t}^{2} v\right\|_{L_{\infty}\left(\mathbb{R}_{+} ; H^{-1}\right)}
$$

Обозначим через $\Theta_{+}^{\text {loc }}$ пространство $\mathscr{F}_{+}^{\text {loc }}$ с локальной $*$-слабой сходимостью в $L_{\infty}^{\text {loc }}\left(\mathbb{R}_{+} ; H^{1}\right), L_{\infty}^{\text {loc }}\left(\mathbb{R}_{+} ; H\right)$ и $L_{\infty}^{\text {loc }}\left(\mathbb{R}_{+} ; H^{-1}\right)$ функций $v, \partial_{t} v$ и $\partial_{t}^{2} v$ соответственно.

Траекторное пространство $\mathscr{K}_{\varepsilon}^{+}$состоит из всех решений $u(x, s)$ уравнения $(3.80)$ из пространства $\mathscr{F}_{+}^{b}$. Аналогично определяется пространство траекторий $\overline{\mathscr{K}}^{+}$для уравнения (3.82). Поскольку $u \in L_{\infty}\left(\mathbb{R}_{+} ; H^{1}\right)$, из (3.81) вытекает, что функция 
$b(x, x / \varepsilon) f(u(x, s)) \in L_{\infty}\left(\mathbb{R}_{+} ; H\right)$ при $u \in \mathscr{K}_{\varepsilon}^{+}\left(\right.$и $\bar{b}(x) f(\bar{u}(x, s)) \in L_{\infty}\left(\mathbb{R}_{+} ; H\right)$ при $u \in \overline{\mathscr{K}}^{+}$соответственно). Следовательно, в уравнениях

$$
\begin{aligned}
& \partial_{t}^{2} u+\gamma \partial_{t} u-\Delta u=-b\left(x, \frac{x}{\varepsilon}\right) f(u(x, t))+g\left(x, \frac{x}{\varepsilon}\right) \equiv F_{\varepsilon}(x, t), \\
& \partial_{t}^{2} \bar{u}+\gamma \partial_{t} \bar{u}-\Delta \bar{u}=-\bar{b}(x) f(\bar{u}(x, t))+\bar{g}(x) \equiv \bar{F}(x, t)
\end{aligned}
$$

правые части $F_{\varepsilon}(x, t)$ и $\bar{F}(x, t)$ принадлежат $L_{\infty}\left(\mathbb{R}_{+} ; H\right)$. Из теоремы ЛионсаМадженеса (см. [20]) следует, что $u, \bar{u} \in C_{b}\left(\mathbb{R}_{+} ; H^{1}\right), \partial_{t} u, \partial_{t} \bar{u} \in C_{b}\left(\mathbb{R}_{+} ; H\right)$. В частности,

$$
\mathscr{K}_{\varepsilon}^{+}, \overline{\mathscr{K}}^{+} \subset C_{b}\left(\mathbb{R}_{+} ; H^{1}\right) \cap\left\{v: \partial_{t} v \in C_{b}\left(\mathbb{R}_{+} ; H\right)\right\}
$$

Легко проверить, что пространства $\mathscr{K}_{\varepsilon}^{+}$при $\varepsilon>0$ и $\overline{\mathscr{K}}^{+}$замкнуты в $\Theta_{+}^{\text {loc }}$. Рассматривается полугруппа трансляций $\{T(t)\}$, действующая на $\mathscr{F}_{+}^{\text {loc }}: T(t) v(x, s)=$ $v(x, s+t), t \geqslant 0$. Доказывается, что для $\mathscr{K}_{\varepsilon}^{+}$и $\overline{\mathscr{K}}^{+}$существует поглощаюшее множество $B_{0}$ вида

$$
B_{0}=\left\{v \in \mathscr{F}_{+}^{b}:\|v\|_{\mathscr{F}_{+}^{b}} \leqslant R_{0}\right\}
$$

где $R_{0}$ не зависит от $\varepsilon$. Уравнения (3.80) и (3.82) имеют траекторные аттракторы $\mathfrak{A}_{\varepsilon}$ и $\overline{\mathfrak{A}}$, которые принадлежат $B_{0}$, компактны в $\Theta_{+}^{\text {loc }}$ и удовлетворяют равенствам

$$
\mathfrak{A}_{\varepsilon}=\Pi_{+} \mathscr{K}_{\varepsilon}, \quad \overline{\mathfrak{A}}=\Pi_{+} \overline{\mathscr{K}},
$$

где $\mathscr{K}_{\varepsilon}$ и $\overline{\mathscr{K}}$ - ядра уравнений $(3.80)$ и (3.82) в пространстве $\mathscr{F}^{b}$ с нормой

$$
\|v\|_{\mathscr{F} b}=\|v\|_{L_{\infty}\left(\mathbb{R} ; H^{1}\right)}+\left\|\partial_{t} v\right\|_{L_{\infty}(\mathbb{R} ; H)}+\left\|\partial_{t}^{2} v\right\|_{L_{\infty}\left(\mathbb{R} ; H^{-1}\right)}
$$

Из результатов предыдушего пункта следует, что $\mathfrak{A}_{\varepsilon} \rightarrow \overline{\mathfrak{A}}$ при $\varepsilon \rightarrow 0+$ в топологическом пространстве $\Theta_{+}^{\text {loc }}$. Мы докажем, что этот предел имеет место в более сильной топологии, если нелинейная функция $f(u)$ удовлетворяет условию (3.81).

Обозначим через $H^{\sigma}=(-\Delta)^{-\sigma / 2} H, \sigma \geqslant 0$, где оператор Лапласа $\Delta$ задан на функциях, удовлетворяющих нулевым граничным условиям $\left.u\right|_{\partial \Omega}=0$. В частности, $H^{0}=H=L_{2}(\Omega), H^{1}=H_{0}^{1}(\Omega), H^{2}=H^{2}(\Omega) \cap H_{0}^{1}(\Omega)$.

Лемма 3.1. Полугруппа $\{T(t)\}$, действующая на $\mathscr{K}_{\varepsilon}^{+}$и $\overline{\mathscr{K}}^{+}$, имеет притягивающее множество

$$
B_{\delta}=\left\{v \in \mathscr{F}_{+}^{b}:\|v\|_{C_{b}\left(\mathbb{R}_{+} ; H^{1+\delta}\right)}+\left\|\partial_{t} v\right\|_{C_{b}\left(\mathbb{R}_{+} ; H^{\delta}\right)} \leqslant R_{\delta}\right\}
$$

при $0 \leqslant \delta<1$. Число $R_{\delta}$ не зависит от $\varepsilon$.

В доказательстве используется лемма 3.4 из [8; гл. VI].

СлЕДСТВИЕ 3.9. Траекторные аттракторы $\mathfrak{A}_{\varepsilon}(\varepsilon>0)$ u $\overline{\mathfrak{A}}$ принадлежат множеству $B_{\delta}, 0 \leqslant \delta<1$. Для любой функиии $u \in \mathscr{K}_{\varepsilon}$ и любой $\bar{u} \in \overline{\mathscr{K}}$

$$
\begin{aligned}
& \|u(t)\|_{H^{1+\delta}}+\left\|\partial_{t} u(t)\right\|_{H^{\delta}} \leqslant R_{\delta} \\
& \|\bar{u}(t)\|_{H^{1+\delta}}+\left\|\partial_{t} \bar{u}(t)\right\|_{H^{\delta}} \leqslant R_{\delta} \quad \text { npu } \quad t \in \mathbb{R} .
\end{aligned}
$$

Сформулируем основной результат. 
ТЕОрема 3.8. Траекторный аттрактор $\mathfrak{A}_{\varepsilon}$ уравнения (3.80) сходится $\kappa$ траекторному аттрактору $\overline{\mathfrak{A}}$ усредненного уравнения $(3.82)$ при $\varepsilon \rightarrow 0+$ в пространствах $C^{\text {loc }}\left(\mathbb{R}_{+} ; H^{1+\delta}\right)$ и $C^{1, \text { lос }}\left(\mathbb{R}_{+} ; H^{\delta}\right)$, где $0 \leqslant \delta<1$. Кроме того, $\mathscr{K}_{\varepsilon}^{+} \rightarrow \overline{\mathscr{K}} n p u \varepsilon \rightarrow 0+{ }_{\text {e }} C^{\mathrm{loc}}\left(\mathbb{R} ; H^{1+\delta}\right) u C^{1, \operatorname{loc}}\left(\mathbb{R} ; H^{\delta}\right)$.

При доказательстве используются неравенства (3.86) и лемма 2.1.

В заключение отметим, что задачи (3.80), (3.83) и (3.82), (3.83) порождают полугруппы $\left\{S_{\varepsilon}(t)\right\}$ и $\{\bar{S}(t)\}$, действующие в пространстве $E=H^{1} \times H$ по формулам:

$$
\begin{aligned}
S_{\varepsilon}(t)\left(u_{0}, u_{1}\right) & =\left(u(t), \partial_{t} u(t)\right), \\
\bar{S}(t)\left(u_{0}, u_{1}\right) & =\left(\bar{u}(t), \partial_{t} \bar{u}(t)\right),
\end{aligned}
$$

которые имеют глобальные $(E, E)$-аттракторы $\mathscr{A}_{\varepsilon}$ и $\overline{\mathscr{A}}$ (см. [8], [7]). Из теоремы 3.8 вытекает

СлЕДСТВИЕ 3.10. Глобальные аттракторы $\mathscr{A}_{\varepsilon}$ u $\overline{\mathscr{A}}$ компактны в $E^{\delta}=$ $H^{1+\delta} \times H^{\delta}, 0 \leqslant \delta<1, u$

$$
\operatorname{dist}_{E^{\delta}}\left(\mathscr{A}_{\varepsilon}, \overline{\mathscr{A}}\right) \rightarrow 0 \quad(\varepsilon \rightarrow 0+) .
$$

\section{А. Доказательство леммы 2.1}

Нам потребуются следующие известные результаты.

Лемма А.1. Пусть $E_{1}, E, E_{0}-$ три банаховых пространства такие, что

$$
E_{1} \Subset E \subset E_{0} .
$$

Тогда для любого $\eta>0$ найдется число $C_{\eta}$ такое, что

$$
\|v\|_{E} \leqslant \eta\|v\|_{E_{1}}+C_{\eta}\|v\|_{E_{0}} \quad \forall v \in E_{1} .
$$

Лемма А.1 доказана в [20; гл. 1, теорема 16.4].

Лемма А.2. Пусть $E_{0}$ и $E-$ банаховъи пространства, $E \subset E_{0}$. Предположим, что функиия $u \in L_{\infty}(\tau, T ; E)$, причем $u(t) \in E_{0}$ для любого $t \in[\tau, T]$. Пусть такжсе функция $\langle u(t), \varphi\rangle$ непрерьвна по $t \in[\tau, T]$, для любого $\varphi \in E_{0}^{*}$ (т.е. $u(t)$ слабо непрерывна из $[\tau, T]$ в $E_{0}$ ). Тогда $u(t) \in E$ при $t \in[\tau, T]$,

$$
\|u(t)\|_{E} \leqslant\|u\|_{L_{\infty}(\tau, T ; E)} \quad \forall t \in[\tau, T]
$$

и и $(t)$ слабо непрерывна из $[\tau, T]$ в $E$.

Доказательство содержится в [20; гл. 3, лемма 8.1].

Докажем лемму 2.1. Пусть $\left\{u_{m}\right\}$ - ограниченная последовательность функций в $W_{\infty, p_{0}}\left(0, T ; E_{1}, E_{0}\right)$. Необходимо проверить, что эта последовательность принадлежит пространству $C([0, T] ; E)$ и предкомпактна в нем. Сначала докажем, что

$$
\left\{u_{m}\right\} \Subset C\left([0, T] ; E_{0}\right) .
$$


По условию последовательность $\left\{u_{m}\right\}$ ограничена в $L_{\infty}\left(0, T ; E_{1}\right)$. Тогда в силу леммы А.2 и (А.3) имеем, что $u_{m}(t) \in E_{1}$ при всех $t \in[0, T]$ и для некоторого числа $C_{1}>0$ выполнено неравенство

$$
\left\|u_{m}(t)\right\|_{E_{1}} \leqslant C_{1} \quad \forall t \in[0, T], \quad \forall m \in \mathbb{N} .
$$

Следовательно, множество

$$
\bigcup_{m}\left\{u_{m}(t): t \in[0, T]\right\} \Subset E
$$

(см. (А.1)), и оно также предкомпактно в $E_{0}$. Так как $\left\{\partial_{t} u_{m}\right\}$ ограничена в $L_{p_{0}}\left(0, T ; E_{0}\right)$, имеем неравенство

$$
\left(\int_{0}^{T}\left\|\partial_{t} u_{m}(s)\right\|_{E_{0}}^{p_{0}} d s\right)^{1 / p_{0}} \leqslant C_{0}
$$

Следовательно, по формуле Ньютона-Лейбница в банаховом пространстве $E_{0}$ получаем:

$$
\begin{aligned}
& \left\|u_{m}\left(t_{2}\right)-u_{m}\left(t_{1}\right)\right\|_{E_{0}}=\left\|\int_{t_{1}}^{t_{2}} \partial_{t} u_{m}(s) d s\right\|_{E_{0}} \\
& \quad \leqslant\left(\int_{t_{1}}^{t_{2}}\left\|\partial_{t} u_{m}(s)\right\|_{E_{0}}^{p_{0}} d s\right)^{1 / p_{0}}\left|t_{2}-t_{1}\right|^{\left(p_{0}-1\right) / p_{0}} \\
& \quad \leqslant\left(\int_{0}^{T}\left\|\partial_{t} u_{m}(s)\right\|_{E_{0}}^{p_{0}} d s\right)^{1 / p_{0}}\left|t_{2}-t_{1}\right|^{\left(p_{0}-1\right) / p_{0} \leqslant C_{0}\left|t_{2}-t_{1}\right|^{\left(p_{0}-1\right) / p_{0}}, \quad p_{0}>1 .}
\end{aligned}
$$

Мы воспользовались здесь неравенством Гёльдера и оценкой (А.6). Следовательно, последовательность $\left\{u_{m}\right\}$ принадлежит $C\left([0, T] ; E_{0}\right)$ и является равностепенно непрерывной. Применяя теорему Арцела-Асколи, заключаем, что $\left\{u_{m}\right\}$ предкомпактна в $C\left([0, T] ; E_{0}\right)$.

Чтобы доказать предкомпактность $\left\{u_{m}\right\}$ в $C([0, T] ; E)$ с помощью теоремы Арцела-Асколи, достаточно проверить равностепенную непрерывность последовательности $\left\{u_{m}\right\}$ в пространстве $C([0, T] ; E)$. (Уже доказано, что значения функций $u_{m}(t)$ принадлежат компактному множеству из $E$, см. (А.4) и (А.5).)

Применим лемму А.1. Зафиксируем любое число $\varepsilon>0$ и возьмем $\eta=\varepsilon /\left(4 C_{1}\right)$ (см. (А.4)). Тогда для некоторого $C_{\eta}>0$ в силу (А.2) получаем:

$$
\begin{gathered}
\left\|u_{m}\left(t_{2}\right)-u_{m}\left(t_{1}\right)\right\|_{E} \leqslant \frac{\varepsilon}{4 C_{1}}\left\|u_{m}\left(t_{2}\right)-u_{m}\left(t_{1}\right)\right\|_{E_{1}}+C_{\eta}\left\|u_{m}\left(t_{2}\right)-u_{m}\left(t_{1}\right)\right\|_{E_{0}} \\
\leqslant \frac{\varepsilon}{4 C_{1}} 2 C_{1}+C_{\eta}\left\|u_{m}\left(t_{2}\right)-u_{m}\left(t_{1}\right)\right\|_{E_{0}} \leqslant \frac{\varepsilon}{2}+C_{\eta} C_{0}\left|t_{2}-t_{1}\right|^{\left(p_{0}-1\right) / p_{0}} .
\end{gathered}
$$

Выбрав $\delta$ так, что

$$
C_{\eta} C_{0} \delta^{\left(p_{0}-1\right) / p_{0}} \leqslant \frac{\varepsilon}{2}
$$

заключаем, что для каждого $m \in \mathbb{N}$ и для любых $t_{1}, t_{2} \in[0, T],\left|t_{2}-t_{1}\right| \leqslant \delta$, выполнено неравенство:

$$
\left\|u_{m}\left(t_{2}\right)-u_{m}\left(t_{1}\right)\right\|_{E} \leqslant \varepsilon .
$$

Следовательно, последовательность $\left\{u_{m}\right\}$ равностепенно непрерывнав $C([0, T] ; E)$, а значит, предкомпактна в $C([0, T] ; E)$. 


\section{Список литературы}

1. Hale J. K., Verduyn Lunel S. M. Averaging in infinite dimensions // J. Integral Equations Appl. 1990. V. 2. № 4. P. 463-494.

2. Ильин А. А. Усреднение диссипативных динамических систем с быстро осциллирующими правыми частями // Матем. сб. 1996. Т. 187. № 5. С. 15-58.

3. Ilyin A. A. Global averaging of dissipative dymanical systems // Rend. Accad. Naz. Sci. XL Mem. Mat. Applicazioni 116. 1998. V. 22. № 1. P. 165-191.

4. Корнфельд И. П., Синай Я. Г., Фомин С. В. Эргодическая теория. М.: Наука, 1980.

5. Левитан Б. М., Жиков В.В. Почти периодические функции и дифференциалњые уравнения. М.: Изд-во МГУ, 1978.

6. Chepyzhov V. V., Vishik M.I. Evolution equations and their trajectory attractors // J. Math. Pures Appl. (9). 1997. V. 76. № 10. P. 913-964.

7. Бабин А. В., Вишик М. И. Аттракторы эволюционных уравнений. М.: Наука, 1989.

8. Temam $R$. Infinite-dimensional dynamical systems in mechanics and physics // Applied Math. Sci. V. 68. New York: Springer-Verlag, 1988.

9. Hale J. K. Asymptotic behaviour of dissipative systems // Math. Surveys Monographs. 25, Amer. Math. Soc., Providence, RI. 1988.

10. Chepyzhov V. V., Vishik M.I. Attractors of non-autonomous dynamical systems and their dimension // J. Math. Pures Appl. (9). 1994. V. 73. № 3. P. 279-333.

11. Chepyzhov V.V., Vishik M.I. Attractors of non-autonomous evolution equations with translation-compact symbols // Oper. Theory Adv. Appl. 1995. V. 78. P. 49-60.

12. Chepyzhov V. V., Vishik M.I. Non-autonomous evolutionary equations with translation-compact symbols and their attractors // C. R. Acad. Sci. Paris Sér. I Math. 1995. V. 321. P. 153-158.

13. Lions J.-L. Quelques méthodes de résolutions des problèmes aux limites non linéaires. Paris: Dunod, Gauthier-Villars, 1969.

14. Chepyzhov V.V., Vishik M.I. Trajectory attractors for 2D Navier-Stokes systems and some generalizations // Topol. Methods Nonlinear Anal. 1996. V. 8. P. 217-243.

15. Chepyzhov V. V., Vishik M.I. Trajectory attractors for reaction-diffusion systems // Topol. Methods Nonlinear Anal. 1996. V. 7. № 1. P. 49-76.

16. Chafee N., Infante $E$. A bifurcation problem for a nonlinear parabolic equation // J. Appl. Anal. 1974. V. 4. P. 17-37.

17. Хенри Д. Геометрическая теория полулинейных параболических уравнений. М.: Мир, 1985.

18. Бесов О. В., Ильин В. П., Никольский С. М. Интегральные представления функций и теоремы вложения. М.: Наука, 1975.

19. Солонников $B . A$. О краевых задачах для линейных параболических систем дифференциалњных уравнений общего вида // Труды МИАН. 1965. Т. 83.

20. Лионс ЖК.-Л., Мадженес Э. Неоднородные граничные задачи и их приложения. М.: Мир, 1971.

Институт проблем передачи информации РАН

E-mail: vishik@iitp.ru; chep@iitp.ru

Поступила в редакцию 27.04 .2000 